\title{
Eine framesemantische Modellierung des juristischen Diebstahl-Begriffs
}

\author{
Detmer Wulf
}

\begin{abstract}
In diesem Beitrag wird eine framesemantische Analyse des Rechtsbegriffs ,Diebstahl' vorgestellt. Die Grundlage für die Analyse bildet zum einen die DiebstahlDefinition in $\S 242$ StGB und zum anderen die Kommentarliteratur zum DiebstahlParagraphen. Während der Gesetzestext vor allem auf die Partizipanten des Wegnahme-Geschehens sowie auf bestimmte Aspekte der täterseitigen Absicht abzielt - Diebstahl ist „Wegnahme“ einer „fremden beweglichen Sache“, mit der Absicht, sie „sich oder einem Dritten rechtswidrig zuzueignen“ - sind die Kommentartexte zum § 242 (unter anderem) um eine Klärung des Wegnahme-Begriffs selbst bemüht, den sie als Bruch fremden und Begründung neuen, nämlich tätereigenen Gewahrsams (an einer Sache) deuten, wobei sie sich ausführlich der Frage widmen, unter welchen Voraussetzungen von Bruch fremden bzw. Begründung neuen Gewahrsams gesprochen werden kann. Im Beitrag soll, zunächst ausgehend von einer im Rahmen des FrameNet-Projekts vorgeschlagenen, Partizipanten-orientierten Analyse, gezeigt werden, wie sich die in den Kommentaren hervorgehobenen Aspekte in eine framesemantische Modellierung einbauen lassen.
\end{abstract}

\section{Einleitung}

Für das Verständnis von Rechtsbegriffen gilt in besonderem Maße, was laut Fillmore Bedeutungsverstehen generell auszeichnet: dass nämlich die Kenntnis der Bedeutung eines Einzelausdrucks immer auch die Kenntnis der konzeptuellen Gesamtstruktur voraussetzt, in die das mit dem Ausdruck assoziierte (Teil-)Konzept eingebettet ist. Dieser holistische Charakter des Bedeutungswissens, den Fillmore im Rahmen seiner „semantics of understanding“ (Fillmore 1985) unter den Begriff des Wissensrahmens (framework of knowledge) fasst (vgl. ebd., $223 \mathrm{f}$.), 
zeigt sich auch in dem Verhältnis der rechtswissenschaftlichen Kommentarliteratur zu den Gesetzestexten des Strafgesetzbuches (StGB) oder des Bürgerlichen Gesetzbuches (BGB), die bemüht ist, über verschiedene Ebenen der begrifflichen Explikation das einzuholen, was in den Gesetzestexten selbst nicht ausgedrückt wird, jedoch im Anwendungsfall mitzuverstehen ist.

So definiert etwa § 242 StGB den Tatbestand des Diebstahls als „Wegnahme“ einer "fremden beweglichen Sache“ mit der Absicht, sie „sich oder einem Dritten rechtswidrig zuzueignen“. Während der Gesetzestext aber lediglich die Partizipanten des Wegnahme-Geschehens - die Sache, den Täter, der (oder die) potenzielle(n) Empfänger - sowie bestimmte Aspekte der täterseitigen Absichten in den Blick nimmt, sind die umfangreichen Kommentartexte zum Diebstahl-Paragraphen (neben vielen weiteren Aspekten) um eine Explikation des WegnahmeBegriffs selbst bemüht, den sie als Bruch fremden und Begründung neuen, nämlich tätereigenen Gewahrsams (an einer Sache) deuten, wobei sie sich ausführlich der Frage widmen, unter welchen Voraussetzungen von Bruch fremden bzw. Begründung neuen Gewahrsams gesprochen werden kann.

Es versteht sich von selbst, dass eine (frame-)semantische Analyse des Verbs stehlen (bzw. des Substantivs Diebstahl), die diese Explikationsebenen mitberücksichtigt, weit über Bedeutungsbeschreibungen hinausgehen muss, die sich - etwa ausgehend von Fillmores (1968) Kasus-Rahmen-Theorie - auf die Angabe der mit dem Verb standardmäßig realisierten (oder ,mitgedachten') ,Mitspieler' (Aktanten) des mit dem Verb assoziierten Verbalgeschehens beschränken. Die Herausforderung für eine framesemantische Analyse des Diebstahlbegriffs besteht somit darin, diese in den Kommentaren entwickelten konzeptuellen Aspekte mit in die Modellierung einzubringen.

Wie eine solche Modellierung aussehen könnte, soll in diesem Beitrag vorgestellt werden. Zunächst folgen jedoch einige einführende Erläuterungen zum Charakter von Rechtsbegriffen im Allgemeinen und - etwas ausführlicher - zum Diebstahl-Begriff im Besonderen (Abschnitt 2 und 3). Im Anschluss (Abschnitt 4) wird begründet, wie sich ein framesemantischer Zugriff auf das Diebstahl-Konzept motivieren lässt, und es werden kurz die Besonderheiten des hier zugrunde gelegten Düsseldorfer Frame-Modells (Petersen 2007; Löbner 2015) erläutert. In den nachfolgenden zwei Abschnitten wird die framesemantische Modellierung vorgestellt, wobei zunächst (Abschnitt 5) der konzeptuelle ,Kern` des Diebstahl- 
Konzepts - der Bruch fremden und die Begründung neuen, tätereigenen Gewahrsams - im Mittelpunkt steht, welcher dann abschließend (Abschnitt 6) durch die noch zu ergänzenden Elemente zu einer vollständigen Modellierung erweitert wird. ${ }^{1}$ Mit in den Blick genommen wird hierbei immer, wie die im DiebstahlParagraphen und in den Kommentaren enthaltenen Definitionen, Explikationen und Verständnisweisen in die Modellierung eingeflossen sind.

\section{Zur Explikationsbedürftigkeit von Rechtsbegriffen}

Anders als das in der angelsächsischen Rechtstradition verwurzelte Fallrecht (case law), in dem sich die Rechtsprechung vornehmlich an vorangegangenen ähnlichen oder vergleichbaren Entscheidungen (Präzedenzfällen) orientiert, basiert die kontinentaleuropäische Rechtsprechung auf kodifiziertem Recht, d. h. Ausgangs- und Orientierungspunkt richterlicher Entscheidungen ist dort primär die in Gesetzen und Verordnungen formulierte rechtliche Norm. Das heißt, dem Normtext (das ist der jeweilige Gesetzesparagraph oder die jeweilige Verordnung) kommt hierbei die Aufgabe zu, bestimmte Sachverhalte rechtlich zu regulieren $^{2}$ oder - bezogen auf das Strafrecht - bestimmte Verhaltensweisen oder Handlungen als Straftatbestände erfassbar und auf dieser Grundlage sanktionierbar zu machen. Vor allem dieser Aspekt trägt zu dem häufig festzustellenden definitorischen Charakter der Normtexte des StGB bei. ${ }^{3}$ Definitorischen Charakter

1 Der in diesem Beitrag vorgestellte Diebstahl-Frame ist in dem zum Sonderforschungsbereich (SFB) 991 „Die Struktur von Repräsentationen in Sprache, Kognition und Wissenschaft“ gehörenden Teilprojekt B05 „Frameanalyse deutscher Rechtsbegriffe“ entwickelt worden, das vom 1.7.2011 bis zum 30.6.2015 von der Deutschen Forschungsgemeinschaft (DFG) gefördert wurde. Die hier vorgestellten Frame-Modellierungen gehören zu den Ergebnissen dieses Projekts, in dem unter der Leitung von Prof. Dr. Dietrich Busse und unter Mitarbeit von Frau Michaela Felden M. A. und dem Verf. Rechtsbegriffe des deutschen Strafrechts (Diebstahl, (mit) Gewalt) sowie des deutschen Zivilrechts (Besitz, Eigentum) unter framesemantischer Perspektive analysiert wurden. Für einen Überblick zu den weiteren Ergebnissen des Projekts siehe Busse (2015a), Busse (2015b) sowie ausführlich Busse/ Felden/Wulf (2018).

2 Als Beispiel kann hier etwa $§ 249$ Abs. 1 BGB dienen, der den Umfang einer Schadensersatzleistung bestimmt: „Wer zum Schadensersatz verpflichtet ist, hat den Zustand herzustellen, der bestehen würde, wenn der zum Ersatz verpflichtende Umstand nicht eingetreten wäre."

3 Natürlich haben nicht alle Paragraphen des StGB definitorischen Charakter oder sind gar Definitionen im streng formalen Sinne. Siehe jedoch beispielsweise die Mord-Definition in $\S 211$ Abs. 2 StGB: „Mörder ist, wer aus Mordlust, zur Befriedigung des Geschlechtstriebs, aus Habgier oder sonst aus niedrigen Beweggründen, heimtückisch oder grausam oder mit gemeingefährlichen Mitteln oder um eine andere Straftat zu ermöglichen oder zu verdecken, einen Menschen tötet." Auf 
hat auch der Diebstahlparagraph des StGB, dessen vollständiger Wortlaut folgendermaßen lautet:

§ 242 StGB Diebstahl: (1) Wer eine fremde bewegliche Sache einem anderen in der Absicht wegnimmt, die Sache sich oder einem Dritten rechtswidrig zuzueignen, wird mit Freiheitsstrafe bis zu fünf Jahren oder mit Geldstrafe bestraft. (2) Der Versuch ist strafbar.

Zwar enthält § 242 StGB keine explizite Diebstahl-Definition, aber Abs. 1 ist doch insofern definitorisch, als sich dort eine Reihe von Angaben darüber finden, welche Bedingungen erfüllt sein müssen, damit eine bestimmte Wegnahme-Handlung als Diebstahl im Sinne von $\S 242$ StGB gelten kann. ${ }^{4}$ Diese Bedingungen beziehen sich zum einen auf das Tatobjekt selbst und zum anderen auf bestimmte Absichten und Einstellungen des Wegnehmenden. Die Tatobjekt-Bedingungen legen zunächst fest, welche Dinge als Tatobjekt eines Diebstahl-Delikts in Frage kommen: Gestohlen werden können laut Abs. 1 nur „Sachen“, die (i) „fremd“ sind, d. h. die nicht zum Allein-Eigentum des Wegnehmenden gehören, und die (ii) „beweglich“ sind, d. h. die über Eigenschaften verfügen, die die Sache überhaupt ,wegnehmbar' machen.

Insofern weist schon die auf das Tatobjekt bezogene Nominalphrase (fremde bewegliche Sache) in mehrfacher Hinsicht über den Normtext, in dem sie enthalten ist, hinaus: erstens, indem sie über das Attribut ,fremd' auf das Eigentumskonzept des Bürgerlichen Gesetzbuches Bezug nimmt, ${ }^{5}$ zweitens, indem sie Wissen darüber voraussetzt, was im rechtlichen Sinne unter einer Sache zu verstehen

der Basis dieser Kriterien ist Mord somit als Untertyp der sog. ,Straftaten gegen das Leben“ ausgezeichnet, für die das deutsche Strafrecht noch eine Reihe weiterer Untertypen definiert, so etwa neben Mord beispielsweise Totschlag (§ 212), Tötung auf Verlangen (§ 216) oder fahrlässige Tötung (§ 222).

4 So könnte man die in Abs. 1 enthaltene ,Definition' etwa so wiedergeben: Diebstahl ist Wegnahme einer fremden beweglichen Sache, in der Absicht, sie sich oder einem Dritten rechtswidrig zuzueignen. Allerdings kennt das deutsche Strafrecht keine Oberklasse der Wegnahme-Delikte, von denen Diebstahl einen Untertyp darstellen würde. Zwar gibt es Deliktstypen wie etwa ,besonders schwerer Fall des Diebstahls' (§ 243 StGB) oder ,Raub' (§ 249 StGB), aber diese stellen lediglich spezifische Fälle des Diebstahl-Delikts dar, das prinzipiell auch ohne die diese Typen auszeichnenden Merkmale vorliegen kann. ,Besonders schwerer Diebstahl liegt u. a. vor, wenn zur Ausführung der Tat in ein Gebäude oder einen verschlossenen Raum eingebrochen wird, wenn jemand ,gewerbsmäßig' stiehlt oder wenn etwa Waffen, Sprengstoff o. ä. gestohlen werden. Raub ist als Diebstahl in Verbindung mit der Anwendung oder Androhung von Gewalt definiert.

5 Vgl. den ersten Satz in § 903 BGB (Befugnisse des Eigentümers): „Der Eigentümer einer Sache kann, soweit nicht das Gesetz oder Rechte Dritter entgegenstehen, mit der Sache nach Belieben verfahren und andere von jeder Einwirkung ausschließen." 
ist, ${ }^{6}$ und drittens, indem sie im konkreten Anwendungsfall u. U. eine Begründung dafür einfordert, dass es sich tatsächlich um eine bewegliche Sache handelt. Denn dass eine Sache beweglich ist, versteht sich nicht unbedingt von selbst! So ist die Einschränkung auf bewegliche Sachen im Fall von Immobilien, d. h. Grundstücken, Gebäuden oder Wohnungen zwar noch unmittelbar einleuchtend: Häuser, Grundstücke usw. sind zumindest nicht im Sinne von $§ 242$ StGB ,wegnehmbar'. Aber wie verhält es sich bei Gras, das ja zunächst fester, d. h. nicht abgetrennter Bestandteil eines Wiesen- oder Weidegrundstücks ist? So ist in einem Urteil gegen einen Schäfer, der seine Schafe vorsätzlich und unbefugt auf einem fremden Grundstück hat weiden lassen (LG Karlsruhe, Urteil vom 21.06.1993 - 8 AK 25/ 93) ${ }^{7}$ die Frage, inwiefern sich abgeweidetes Gras als bewegliche Sache begreifen lässt, für die Subsumtion seiner Tat unter den Straftatbestand des Diebstahls durchaus relevant gewesen. Die Begründung lautet dort folgendermaßen:

Der Tatbestand des Diebstahls ist dadurch erfüllt, daß die Schafe des Angekl. das auf fremden Grundstücken wachsende Gras sowie Klee abgerissen und gefressen haben. Ursprünglich handelte es sich bei dem Gras nicht um eine bewegliche Sache, da es fest mit dem Boden verbunden war. Es sind jedoch auch Teile von unbeweglichen Sachen als beweglich anzusehen, wenn sie losgelöst und beweglich gemacht werden. [...] Bei dem Vorgang des Abfressens wurden die Halme zunächst vom Boden abgetrennt und dadurch zu beweglichen Sachen i. S. des § 242 StGB. (NStZ 1993, 543)

Was hier nur in groben Zügen am Beispiel der Tatobjekt-Bedingungen erläutert wurde, trifft auch auf andere zentrale Begriffe in § 242 StGB zu. Auch für sie gilt:

6 Was im rechtlichen Sinne eine Sache ist, bestimmt $\S 90$ BGB (Begriff der Sache) - der allerdings nur die knappe Auskunft gibt: „Sachen im Sinne des Gesetzes sind nur körperliche Gegenstände." Was der Normtext somit nicht leistet (und prinzipiell auch nicht leisten kann), ist die Explikation seiner eigenen Explikationsbegriffe. Wer also wissen möchte, was rechtlich zu den körperlichen Gegenständen gezählt wird (und was nicht), ist auf die entsprechenden Kommentare (und/oder auf damit befasste Rechtsurteile oder Entscheidungen, die mit in die Kommentierungen eingeflossen sind) angewiesen; vgl. etwa die Auskunft des Münchener Kommentars (MüKo) zum § 90 BGB, Randnummer (Rn) 1: „Der im Gesetz nicht definierte Oberbegriff, Gegenstände umfasst alle individualisierbaren, vermögenswerten Objekte und Güter, über die Rechtsmacht im Sinne von Herrschafts- oder Nutzungsrechten ausgeübt werden kann." Nicht stehlbar sind darum Personen, die zwar körperlich, aber nicht Sachen, sondern „Rechtssubjekte“ sind (vgl. MüKo § 90 BGB, Rn 2). Keine Sache im Sinne des $§ 90$ BGB ist etwa auch elektrischer Strom, da ihm die Eigenschaft der Körperlichkeit abgesprochen wird (vgl. MüKo § 90 BGB, Rn 24) - was es im Strafrecht notwendig gemacht hat, die rechtswidrige Zueignung elektrischer Energie als eigenen, vom Diebstahl zu unterscheidenden Straftatbestand zu konstituieren (siehe § 248c StGB: Entziehung elektrischer Energie).

7 In Auszügen veröffentlicht in: Neue Zeitschrift für Strafrecht (NStZ) 1993, 543 f. 
Nicht der Normtext allein kann leisten, dass ein bestimmtes ,Realgeschehen' unter den im Normtext benannten Straftatbestand subsumierbar ist. Der Normtext selbst - und die in ihm enthaltenen rechtlich relevanten Begriffe - setzt einen Wissens- und Verstehenshorizont voraus, der zum einen auf andere Normtexte bezogen ist und zum anderen Verstehensweisen vorangegangener Urteile oder obergerichtlicher Grundsatzentscheidungen umfasst, welche in die Kommentarliteratur eingeflossen sind und auf diese Weise das Verständnis des Normtexts und damit auch wiederum seine Anwendung in konkreten Entscheidungsfällen - prägen und lenken.

Ebenso explikationsbedürftig wie der Begriff der Sache ist der Begriff der $\mathrm{Zu}-$ eignung, der im Zusammenhang mit $§ 242$ StGB insofern von Bedeutung ist, als er auf die für den Diebstahl-Begriff tatbestandsrelevanten Täterabsichten abstellt: Der Täter nimmt die Sache in der Absicht weg, sie sich (oder einem Dritten) rechtswidrig zuzueignen. (Was unter ,Zueignung' zu verstehen ist und welche Konsequenzen sich daraus für das Diebstahl-Konzept ergeben, werde ich im nächsten Abschnitt erläutern.)

Und auch das im Normtext enthaltene Verb wegnehmen wird in der Kommentarliteratur zum § 242 StGB umfassend expliziert: Eine Handlung, die unter Diebstahl subsumierbar ist, ${ }^{8}$ zeichnet sich dadurch aus, dass durch Bruch fremden und Begründung neuen Gewahrsams eine „Gewahrsamsverschiebung“ vom Bestohlenen auf den Wegnehmenden stattfindet. Stellvertretend für viele andere ähnlich lautende Kommentarstellen sei hier Randnummer (Rn) 22 in Schönke/Schröders Strafgesetzbuch-Kommentar wiedergegeben: ${ }^{9}$

Die Tathandlung [des Diebstahls] besteht in der Wegnahme der Sache: durch Bruch fremden und Begründung neuen (idR eigenen) Gewahrsams [...]. Aufgrund der dafür erforderlichen Gewahrsamsverschiebung vom Opfer zum Täter kommt

8 Die Subsumtion einer Handlung unter den Begriff des Diebstahls erfolgt somit auf der Basis bestimmter, für Diebstahl-Handlungen notwendig geltender Tatbestandsmerkmale. Insofern enthält $\S 242$ StGB zumindest implizit eine Definition, die über den Begriff der Wegnahme dem ,klassischen' Muster der Begriffsdefinition auf der Basis von genus proximum und differentia specifica nahekommt: Diebstahl ist Wegnahme einer fremden beweglichen Sache, in der Absicht, sie sich (oder einem Dritten) rechtswidrig zuzueignen.

9 Es ist üblich, die Kommentarstellen durch Randnummern (Rn) anzugeben. Die Randnummernzählung ist immer auf den jeweiligen kommentierten Paragraphen bezogen und beginnt mit dem nachfolgenden Paragraphen wieder neu. Die Identifizierung der zitierten Kommentarstelle erfolgt also über die Angabe des kommentierten Paragraphen und über die Randnummer, die der zitierten Stelle zugeordnet ist. 
dem Gewahrsamsbegriff eine Schlüsselfunktion zur Bestimmung der Wegnahme zu [...]. (Schönke/Schröder § 242 StGB, Rn 22)

Durch die Konzeptualisierung von Wegnahme als Bruch und Begründung (die Wegnahme bewirkt eine Gewahrsamsverschiebung vom Bestohlenen auf den Wegnehmenden) kommt ein weiterer klärungsbedürftiger Begriff ins Spiel: der Begriff des Gewahrsams. Dieser wird darum in einem nächsten Schritt expliziert als „tatsächliches Herrschaftsverhältnis zwischen einer Person und einer Sache [...], das von einem Herrschaftswillen [...] getragen ist" (Schönke/Schröder $§ 242$ StGB, Rn 23).

\section{Rechtsbegriff \\ 2. Definition in $§ 242$ \\ 3. rechtsdogmatische Definition \\ 4. rechtsdogmatische Definition \\ 5. rechtsdogmatische Definition \\ 6. subsumierter Sachverhalt}
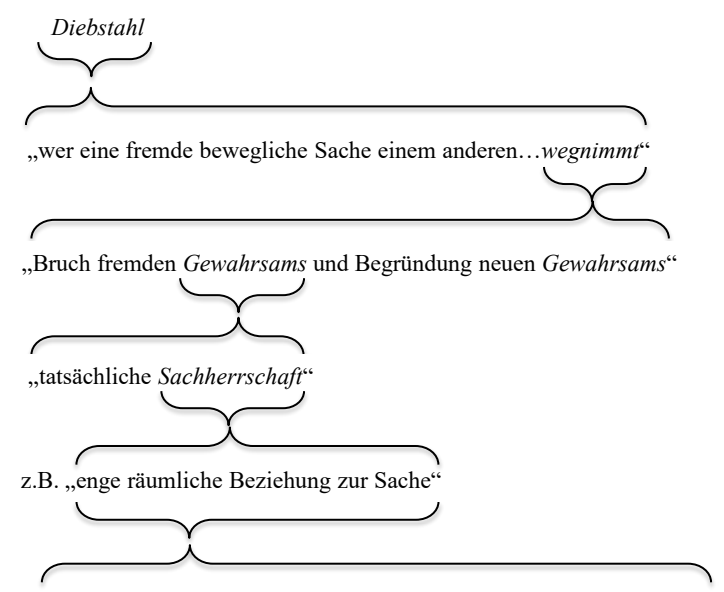

z.B. ,der Wohnungbesitzer hat Gewahrsam an den in seiner Wohnung befindlichen Gegenständen, auch wenn er abwesend ist“"

Abb. 1: Explikationsstufen von wegnehmen in $§ 242 \mathrm{StGB}$, orientiert an Busse (2002)

Gewahrsam ist also zu verstehen als „Sachherrschaftsverhältnis“, das sich darin auszeichnet, dass „der unmittelbaren Verwirklichung des Einwirkungswillens auf die Sache keine Hindernisse entgegenstehen [...]“ (ebd., Rn 25), was u. a. dadurch gewährleistet ist, dass eine „enge räumliche Beziehung“ zwischen der Person und der Sache besteht (ebd.), etwa wenn sich die Sache in der Wohnung der Person befindet - welche nach herrschender Meinung aber auch dann noch fortbesteht, wenn sich die Person vorübergehend nicht in ihrer Wohnung aufhält (ebd. Rn 
26). Wie Abb. 1 zeigt, lassen sich auf diese Weise bis zu sechs Explikationsstufen unterscheiden. Abb. 1 liefert natürlich noch kein vollständiges Bild: Zum einen bleibt die auf Stufe 3 gewählte Fortführung des Explikationspfads auf den Gewahrsamsbegriff beschränkt und lässt die Explikation der Begriffe ,Bruch“ und ,Begründung' unberücksichtigt. Und zum anderen wird auf der Ebene der Sachherrschaftsexplikation (Stufe 5), ebenso wie auf der Ebene der üblicherweise unter Sachherrschaft subsumierten ,realweltlichen' Sachverhalte (Stufe 6), lediglich ein Aspekt bzw. ein typisches Beispiel genannt.

\section{Bruch und Begründung vs. Zueignung: Begriffliche Merkmale des Diebstahl-Delikts}

$\S 242$ StGB Abs. 1 bestimmt Diebstahl zunächst als Wegnahme einer fremden beweglichen Sache. Die Wegnahme-Handlung selbst ist in der Kommentarliteratur als „Bruch fremden und Begründung neuen [in der Regel tätereigenen] Gewahrsams“ definiert (siehe oben, Schönke/Schröder § 242 StGB, Rn 22). Des Weiteren gibt Abs. 1 bestimmte täterseitige Absichts- und Wissensaspekte an: Der Täter nimmt die Sache weg, um sie „sich oder einem Dritten rechtswidrig zuzueignen“. Der Wegnahme-Handlung liegt somit (i) eine Zueignungsintention zugrunde und diese Zueignungsintention ist (ii) von dem Wissen begleitet, dass die Sache dem Täter rechtmäßig nicht zusteht. ${ }^{10}$ Darüber hinaus wird in Abs. 2 noch die (konzeptuell zunächst relativ harmlos erscheinende) Auskunft gegeben, dass auch der Versuch strafbar ist.

Klärungsbedürftig ist jedoch nicht nur, wann bzw. unter welchen Bedingungen von Bruch und (im Anschluss daran) von Begründung gesprochen werden kann - sondern auch, wie das Verhältnis von Begründung und Zueignung zu verstehen ist. Zwei Deutungsmöglichkeiten bieten sich hier an: Eine wäre, Begründung und Zueignung als zeitlich und logisch zusammenfallend aufzufassen, so-

\footnotetext{
${ }^{10}$ Das Wissen um die Unrechtmäßigkeit der Zueignung ist darum notwendig für die Subsumtion einer Wegnahme-Handlung unter den Diebstahl-Tatbestand. Nimmt der Wegnehmende etwa irrtümlich an, dass die Zueignung aus bestimmten Gründen nicht unrechtmäßig ist (etwa weil er die Sache für seine eigene hält oder denkt, dass sie herrenlos ist, vgl. MüKo § 242 StGB, Rn 153), so ist der Aspekt der Unrechtmäßigkeit auch nicht Bestandteil seines Vorsatzes, sich die Sache zuzueignen.
} 
dass im Fall erfolgreicher Gewahrsamsbegründung auch die erfolgreich vollzogene Zueignung angenommen werden müsste. Die andere Möglichkeit ist, Begründung und Zueignung konzeptuell voneinander zu trennen, mit der Folge, dass die Wegnahme-Handlung selbst - die sich ja laut Kommentarliteratur aus dem Bruch fremden und der Begründung eigenen Gewahrsams zusammensetzt von der Frage der erfolgreichen Zueignung zu lösen wäre. Das heißt, nach dieser Deutung ist die (erfolgreiche) Begründung tätereigenen Gewahrsams prinzipiell unabhängig davon, ob am Ende auch die Zueignung erfolgreich vollzogen werden konnte. Die Rechtsprechung hat sich für die letztere Deutungsvariante entschieden. Der Grund für diese aus der Laienperspektive vielleicht nicht unmittelbar einleuchtende Verstehensweise ist, dass sich so eine Regelung für die in der Rechtsprechungspraxis außerordentlich wichtige Frage formulieren lässt, ab wann von vollendetem Diebstahl gesprochen werden kann. Denn da Abs. 2 festhält, dass auch versuchter Diebstahl strafbar ist, muss ebenfalls geklärt werden, ob ein Wegnahme-Fall noch als versucht oder schon als vollendet anzusehen ist. So könnte man etwa dafür argumentieren, dass eine Wegnahme-Handlung, bei der der Täter ,auf frischer Tat' ertappt wird, unter versuchten Diebstahl zu subsumieren sei, da die Zueignung in einem solchen Fall ja nicht erfolgreich vollzogen worden ist. Die Rechtsprechung hat sich gegen diese Sichtweise entschieden. Diebstahl gilt vielmehr schon dann als vollständig vollzogen, wenn „der Dieb auf frischer Tat betroffen wird“, weshalb „nicht erst das Fortschaffen oder gar Bergen des Diebesguts den Zeitpunkt der Vollendung markieren“ kann (Leipziger Kommentar (LK) § 242 StGB, Rn 48).

Diese Bestimmung scheint auf den ersten Blick etwas stipulativ zu sein, lässt sich aber nach der Auffassung des Leipziger Kommentars klar dem Normtext entnehmen: Zueignung sei darum von der Wegnahme „streng [...] zu unterscheiden“, weil sie „nach dem Gesetzeswortlaut [!] nicht Tathandlung des Diebstahls ist“, sondern lediglich „beabsichtigt, nicht aber eingetreten sein muss“ (LK § 242 StGB, Rn 51; siehe auch Abb. 2). Zueignung ist laut Fischer (§ 242 StGB, Rn 33) „die Begründung des Eigenbesitzes unter Ausschluss des Berechtigten mit dem Willen, wie ein Eigentümer über die Sache zu verfügen“. Aber ob es im Anschluss an den Diebstahl „tatsächlich zu einer Zueignung kommt, ist [...] unerheblich“ (Fischer § 242 StGB, Rn 32). Diebstahl ist darum ein Delikt mit „überschießender 
Innentendenz“ und ein sog. „kupiertes Erfolgsdelikt“. Mit ,überschießender Innentendenz' ist gemeint, dass der Aspekt der Zueignungsabsicht notwendiger subjektiver Bestandteil der Wegnahme-Handlung ist, ohne den sich Wegnahme im Sinne von § 242 StGB nicht denken lässt, der aber prinzipiell unabhängig davon ist, ob die Zueignung am Ende tatsächlich erfolgreich vollzogen werden konnte. Eben dies macht Diebstahl zu einem „kupierten Erfolgsdelikt“: Denn egal ob die Zueignung erfolgreich war oder nicht - es handelt es sich in jedem Fall um Diebstahl. ${ }^{11}$

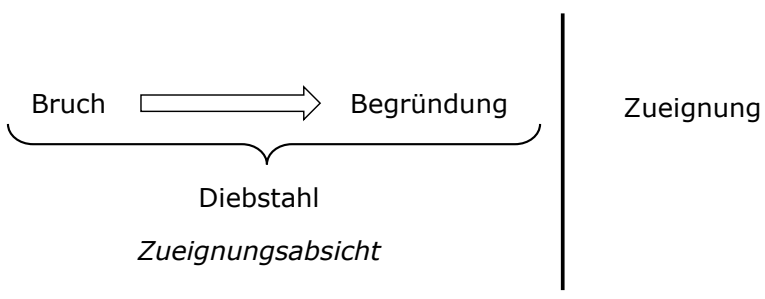

Abb. 2: Wegnahme vs. Zueignung

Der Sinn dieser Konstruktion besteht somit darin, von Diebstahl schon ab dem Zeitpunkt sprechen zu können, an dem der Gewahrsamsübergang abgeschlossen ist, d. h. wenn der neue, tätereigene Gewahrsam begründet (worden) ist. Wird der Täter im Moment der Gewahrsamsbegründung oder direkt im Anschluss daran auf frischer Tat ertappt, so ist der Diebstahl nicht als versucht, sondern als schon vollendet aufzufassen, ohne dass die Zueignung erfolgt ist. „Kupiert“ ist der Erfolg für den Täter dann in dieser Hinsicht: Der Täter hat nicht erreicht, was er mit der Tat eigentlich erreichen wollte, nämlich im Anschluss daran „wie ein Eigentümer über die Sache [...] verfügen“ zu können (siehe oben, Fischer $§ 242$ StGB, Rn 33).

Die Gewahrsamsbegründung besteht demgegenüber lediglich in der Herstellung aktueller Sachherrschaft, für die nicht erforderlich ist, dass sie längere Zeit andauert:

${ }^{11}$ Darum kann z. B. Mord niemals ein in diesem Sinne „kupiertes“ Erfolgsdelikt sein. Denn um eine Tathandlung unter den Straftatbestand ,Mord' subsumieren zu können, muss die Tat ,erfolgreich vollzogen worden sein, d. h. das Opfer muss tot sein. Wäre das Opfer nicht tot, hätte der Täter keinen Mord, sondern lediglich einen Mordversuch begangen. 
Neuer Gewahrsam ist begründet, wenn der Täter tatsächliche Herrschaft über die Sache erlangt und die Herrschaft - wenn auch nur vorübergehend [...] - unbehindert ausüben kann, während der frühere Gewahrsamsinhaber zur Rückerlangung der Sachherrschaft die Verfügungsgewalt des Täters erst beseitigen müsste. [...] Gesichert muss der Gewahrsam noch nicht sein. (LK § 242 StGB, Rn 93 f.)

Voraussetzung für die Gewahrsamsbegründung ist der Bruch, d. h. die „Aufhebung" bestehenden Gewahrsams durch den Täter:

„Weg“nehmen kann nur, wer den bestehenden Gewahrsam des ursprünglichen Gewahrsamsinhabers aufhebt. Entscheidend ist, dass dieser nicht mehr ohne weiteres an die Sache herankommen und seine Herrschaft ohne Beseitigung der faktischen Verfügungsgewalt des Diebes nicht mehr wahrnehmen kann. (LK § 242 StGB, Rn 87)

Ein weiterer entscheidender Punkt ist hierbei, dass die Wegnahme „gegen oder ohne den Willen des [Gewahrsams-]Berechtigten erfolgt, es also am so genannten tatbestandsausschließenden Einverständnis fehlt." (Joecks, § 242 StGB, Rn 10). $\mathrm{Zu}$ beachten ist hier die Redeweise „gegen oder ohne den Willen des Berechtigten“. Für die Annahme, dass das „tatbestandsausschließende Einverständnis“ fehlt, ist nämlich nicht notwendig, dass der Gewahrsamsberechtigte den Bruch bemerkt, vielmehr reicht schon aus, dass er sein Einverständnis in den Gewahrsamsübergang nicht zu erkennen gegeben hat. Im ersteren Fall (wenn er die Wegnahme bemerkt) geschieht der Bruch gegen seinen Willen, im letzteren Fall (wenn er die Wegnahme nicht bemerkt) geschieht der Bruch ohne seinen Willen. Für Wegnahme-Fälle gilt demnach: Seitens des Geschädigten besteht kein erkennbares Einverständnis in den Gewahrsamsverlust an dem Tatobjekt, für das er Gewahrsamsberechtigung hat (d. h. er hätte es nicht gegeben, wenn er den Bruch bemerkt hätte). Das fehlende Einverständnis ist darum ein weiteres notwendiges Tatbestandmerkmal: Würde seitens des Gewahrsamsberechtigten Einverständnis bestehen, ließe sich der Gewahrsamsverlust nicht mehr auf einen Bruch zurückführen, womit dann auch nicht mehr von Diebstahl gesprochen werden könnte. ${ }^{12}$

12 Dennoch könnte auch bei vorausgesetztem Einverständnis in den Gewahrsamsübergang täterseitig die Absicht bestehen, sich die Sache rechtswidrig zuzueignen, etwa wenn der Täter beabsichtigt, eine Sache, die ihm anvertraut wurde, nicht zurückzugeben. Eine solche Tatkonstellation fällt nicht unter den Straftatbestand des Diebstahls, sondern unter den der Unterschlagung (§ $246 \mathrm{StGB}$ ). Allerdings ist Unterschlagung nicht auf das Einverständnis-Merkmal angewiesen. Wer bspw. eine verlorengegangene Geldbörse mit einen größeren Geldbetrag findet und diese nicht ins Fundbüro bringt, macht sich ebenfalls der Unterschlagung schuldig. Zwar besteht hier seitens des rechtmäBigen Besitzers sicherlich kein Einverständnis darüber, dass der Finder das Geld behält, aber da 
Fassen wir zusammen: Wegnahme im Sinne von $\S 242$ StGB besteht im Bruch fremden und in der Begründung neuen, in der Regel tätereigenen Gewahrsams. Die Wegnahme-Handlung ist begleitet (bzw. motiviert) von der Absicht des Täters, sich die Sache zuzueignen, wobei er sich über die Rechtswidrigkeit der Zueignung im Klaren ist. Bruch setzt voraus, dass kein Einverständnis des Gewahrsamsberechtigten in den Gewahrsamsübergang besteht. Die Begründung ist erfolgt (und die Wegnahme-Handlung damit vollendet), sobald der Täter - wenn auch nur vorübergehend - Sachherrschaft über die Sache erlangt hat.

Die Entscheidung, Wegnahme mit der Gewahrsamsbegründung als vollendet aufzufassen, hat entscheidende Auswirkungen darauf, was als versuchter Diebstahl gelten kann. Da Zueignung auf diese Weise so konzeptualisiert wird, dass sie der Wegnahme zeitlich nachgelagert ist, lassen sich Fälle ,missglückter ${ }^{`} \mathrm{Zu}$ eignung (etwa wenn der Täter , auf frischer Tat ertappt' wird) nicht als Fälle versuchten Diebstahls begreifen. Dies hat zur Folge, dass versuchter Diebstahl zeitlich vor der Begründung verortet werden muss (vgl. bspw. MüKo § 242 StGB, Rn 166: „Im Stadium des Versuchs befindet sich der Täter bis zur Begründung neuen Gewahrsams“) und somit Fälle betrifft, in denen die Begründung aufgrund ,missglückter ' Bruch-Aktivitäten nicht erfolgt ist (für Beispiele siehe LK § 242 StGB, Rn 193 f. sowie Fischer $§ 242$ StGB, Rn 55 f.). ${ }^{13}$

\section{Das Wegnahme-Konzept aus framesemantischer Sicht}

Frametheoretische Ansätze implizieren eine verstehensorientierte und eine konzeptorientierte Perspektive. Die erstere Perspektive hebt die entscheidende Rolle vorausgesetzten, nicht explizit gemachten Wissens für das Verstehen von Texten, Äußerungen oder Begriffen hervor. Wie wir gesehen haben, setzt auch das vollständige Verständnis eines Normtextes wie § 242 StGB Wissen darüber voraus,

sein Gewahrsam an der Geldbörse und dem darin enthaltenen Geld zu dem Zeitpunkt, an dem der Finder die Geldbörse an sich nimmt, schon nicht mehr besteht, liegt hier auch kein Bruch vor.

13 Zum versuchten Diebstahl werden allerdings auch Fall-Konstellationen gezählt, in denen der Täter nicht weiß, dass der Eigentümer die Wegnahme insgeheim geschehen lassen will. Dies ist etwa bei der sogenannten ,Diebesfalle‘ der Fall. Von Diebesfalle spricht man, wenn die Wegnahme bewusst zugelassen wird, um den Täter im Anschluss als Dieb überführen zu können, was u. U. erst nach dem Zeitpunkt der Gewahrsamsbegründung geschieht. Laut LK § 242 StGB, Rn 127 liegt in solchen Konstellationen Versuch vor, weil aufgrund des vorhandenen Einverständnisses in den Gewahrsamsübergang kein Bruch stattgefunden hat. 
was im rechtlichen Sinne eine Sache ist, was unter Zueignung verstanden wird und noch vieles andere mehr. Die letztere Perspektive zielt demgegenüber auf das Wissen selbst ab, wobei in frametheoretischen Ansätzen von der These ausgegangen wird, dass dieses (verstehensrelevante) Wissen in einem kognitiven Format repräsentiert ist, das bestimmte allgemeine Strukturmerkmale aufweist. Diese zwei Perspektiven sind jedoch nicht voneinander zu trennen. Die erstere ist vielmehr bedingt durch die letztere: Voraussetzung für das Verstehen ist, auf schon vorhandene Wissensrepräsentationen zurückgreifen zu können, die dann auf das zu Verstehende ,angewendet" werden. Diesen Mechanismus hat schon Minsky (1975) beschrieben:

When one encounters a new situation [...] one selects from memory a structure called a frame. This is a remembered framework to be adapted to fit reality by changing details as necessary. A frame is a data-structure for representing a stereotyped situation. (Minsky 1975, 212)

Die einen Frame konstituierende „Daten-Struktur“ beschreibt Minsky als ein Netzwerk aus „nodes“ und „relations“, das sich als ein ,Gerüst” stabiler, standardmäßig mit der repräsentierten Situation assoziierter Strukturelemente denken lässt, deren „slots“ im ,Anwendungsfall“ dann jeweils situationsspezifische „filler“ zugewiesen werden (ebd.). ${ }^{14}$

Auch die Wegnahme- bzw. Diebstahl-Situation lässt sich so zunächst als abs-

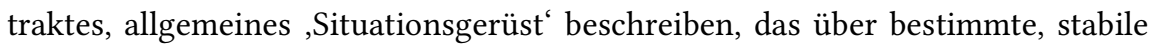
Strukturelemente verfügt: etwa, dass die Wegnahme immer von irgendjemandem ausgeführt wird (dem Täter), des Weiteren, dass es immer etwas gibt, das weggenommen wird (die Sache), sowie, dass es immer jemanden gibt, dem die Sache weggenommen wird (den Geschädigten). Vor diesem Hintergrund ist es dann naheliegend, die aufgelisteten Elemente (den Täter, das Tatobjekt bzw. die Sache, den Geschädigten) als die standardmäßig involvierten ,Partizipanten“ oder ,Mitspieler' des mit dem Verb wegnehmen (bzw. stehlen) assoziierten Verbalgeschehens aufzufassen. Dies ist eine Idee, die, inspiriert von Fillmores (1968) „case frame“-Ansatz, auch dem Frame-Verständnis des lexikalisch/semantisch orientierten, ebenfalls von Fillmore initiierten FrameNet-Projekts zugrunde liegt:

\footnotetext{
14 Vgl. ebenfalls Minsky (1986, 244 f.): „A frame is a sort of skeleton, somewhat like an application form with many blanks or slots to be filled."
} 
The word frame in this context is used to refer to a schematic representation of speakers' knowledge of the situations or states of affair that underlie the meanings of lexical items. The named components of a frame, called frame elements (FEs), stand for the participants, props, phases, and parts of the kinds of situations named by the frame. (Fillmore 2007, 130)

Bezogen auf das Verb stehlen heißt dies: Das der Bedeutung von stehlen zugrunde liegende (Frame-)Konzept setzt sich zusammen aus den mit der Wegnahme- bzw. Diebstahl-Situation standardmäßig assoziierten Partizipanten (Ausführender, Betroffener und (gestohlene) Gegenstände) sowie aus bestimmten lokalen und/oder instrumentalen Informationen. Ein Ausdruck wie stehlen ist darum laut FrameNet assoziiert mit Situationen ,in which a PERPETRATOR takes GOODS from a VICTIM or a SOURCE" ${ }^{15}$ Die in dieser Beschreibung aufgelisteten semantischen RollenBezeichnungen - bzw. in der FrameNet-Terminologie: Frame-Elemente (vgl. Fillmore 2007, 129) - stellen die sogenannten „core elements“ dar, die im FrameNetEintrag für theft wie folgt bestimmt sind: ${ }^{16}$

- GoODS: alles das, was weggenommen werden kann

- Perpetrator: die Person, die die Sachen wegnimmt

- SOURCE: der Ort, an dem die Sache gelegen hat, bevor sie weggenommen wurde

- Victim: derjenige, der die Sache besessen hat, bevor sie vom Täter weggenommen wurde

Alle diese Frame-Elemente sind Bestandteile des mit stehlen (oder mehr oder weniger synonymen Ausdrücken wie klauen, stibitzen, entwenden etc.) assoziierten schematischen Wissens und können in Sätzen, die diese Verben enthalten, in verschiedenen Kombinationen realisiert sein, entweder durch Argumente oder z. T. auch durch attributive Erweiterungen innerhalb von Argument-Konstituenten:

(1) Arno stahl die Uhr (aus Utes Handtasche). Arno stahl (Ute) die Uhr (aus der Handtasche).

${ }^{15}$ Diese Definition findet sich in der FrameNet-Datenbank (https://framenet.icsi.berkeley.edu) unter dem Eintrag für theft (zuletzt abgerufen am 8.6.2015).

16 Des Weiteren werden für stehlen noch eine Reihe von „non core elements“ aufgelistet, zu denen laut FrameNet u. a. auch die Instrumental-Rolle (MEANS) zählt. 
Die Beispiele in (1) orientieren sich grob an den Beispielen im FrameNet-Eintrag zu theft. Zunächst fällt auf, dass die jeweilige ,Rolle“ der realisierten „core elements“ (PERPETRATOR, SOURCE, VicTIM etc.) zumindest nicht vollständig die Semantik (oder den Typ) der verwendeten Ausdrücke determiniert, durch die sie repräsentiert sind. So ist etwa die Handtasche nicht per se SOURCE, sondern könnte ebenso gut auch Tatobjekt sein. ${ }^{17}$ Und nichts kommt Arno bzw. Ute als Eigenschaft zu, das sie prinzipiell als PERPETRATOR bzw. VICTIM ausweist. Ihre jeweilige Rolle wird ihnen vielmehr durch das Verb stehlen zugewiesen, das ihnen zunächst auf einer wesentlich abstrakteren Ebene die Agens- bzw. Patiens-Rolle zuweist, die dann - auf der Basis der Semantik von stehlen (bzw. des mit dem Ausdruck assoziierten konzeptuellen Wissens) - jeweils als PERPETRATOR bzw. VICTIM spezifiziert wird. Bildet man auf diese Struktur Minskys slot/filler-Unterscheidung ab, so lassen sich Arno, Ute, die Uhr usw. als filler für die jeweiligen slots PERPETRATOR, GOODS, VICTIM etc. auffassen. Insofern stellen die Sätze in (1) also Repräsentationen von konkreten Diebstahl-Situationen dar, während die ,allgemeine' Frame-Struktur der Diebstahl-Situationen zunächst allein durch die slots repräsentiert wird und die jeweiligen ,Füllungen“ somit als Instantiierungen aufzufassen sind, d. h. die in der konkreten Diebstahl-Situationen involvierten Partizipanten repräsentieren.

Es ist aber ebenso möglich, die slot/filler-Unterscheidung auf die Ebene des ,allgemeinen' Diebstahl-Konzepts anzuwenden, ohne dabei auf konkrete Diebstahl-Instantiierungen Bezug zu nehmen. Dies ist für die framesemantische Modellierung darüber hinaus sogar notwendig, da sich die ,allgemeinen' strafrechtlich relevanten Aspekte des Diebstahl-Konzepts gar nicht auf die Ebene konkreter Instantiierungen ,herunterbrechen' lassen. Dies gilt schon für das Tatobjekt: Für das Wegnahme-Konzept in $\S 242$ StGB ist nicht relevant, ob es sich um eine Uhr, eine Geldbörse oder um sonst etwas handelt - relevant ist vielmehr die Klärung der Frage, über welche Eigenschaften ein Gegenstand generell verfügen muss, um

\footnotetext{
17 Vgl. noch einmal die Definition in FrameNet: „SOURCE is the initial location of the goods, before they change location." Zwar lässt sich eine Handtasche durchaus als Ort konzeptualisieren, aber auch dies macht die Handtasche noch nicht zur SOURCE. Auch ein Ort ist nicht per se SOURCE. Ebenso wenig erfordert die SoURCE-Lesart, dass die Handtasche innerhalb eines Direktiv-Komplements (aus der Handtasche) realisiert wird, denn die ,initial location“ könnte genauso gut durch einen Attributsatz angegeben werden: Arno stahl die Uhr, die sich in Utes Handtasche befand. Und auch hier ist die „location“ der Uhr erst in Verbindung mit dem Verb stehlen und den anderen am Wegnahme-Geschehen involvierten Partizipanten als SOURCE deutbar.
} 
unter die Kategorie ,Tatobjekt einer Diebstahl-Handlung' subsumiert werden zu können. So legt $\S 242$ StGB in Bezug auf das Tatobjekt fest, dass es sich um eine fremde bewegliche Sache handeln muss. Filler der Partizipanten-Ebene wäre hier also nicht irgendeine Uhr, Geldbörse oder sonst etwas, sondern der allgemeine Begriff ,Tatobjekt', für den es dann im nächsten Schritt - auf der Ebene des TeilKonzepts ,Tatobjekt ${ }^{\text {' }}$ - gilt, die für das Tatobjekt notwendigen allgemeinen Merkmale zu benennen. Die im Normtext und in den Kommentaren enthaltenen Informationen sind in Abb. 3 grafisch modelliert.

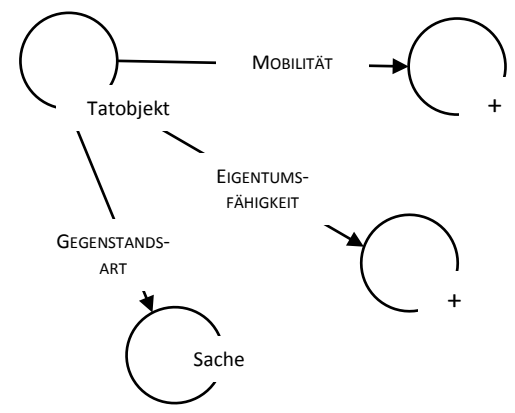

Abb. 3: Teil-Frame, Tatobjekt‘

Die grafische Modellierung in Abb. 3 (sowie die anderen Frame-Modellierungen) folgt den Konventionen des im DFG-Sonderforschungsbereich 991 „Die Struktur von Repräsentationen in Sprache, Kognition und Wissenschaft" entwickelten Frame-Modells. ${ }^{18}$ Die ,klassische slot/filler-Terminologie wird in diesem Modell durch die Unterscheidung von Attributen und Werten ersetzt, wobei die Werte grafisch durch Kreise (,Knoten') und die Attribute durch Pfeile repräsentiert sind. Die Modellierung ist so zu verstehen, dass den Attributen jeweils ein bestimmter, in dem jeweiligen Wert-Knoten benannter Wert zugeordnet ist, wobei die Attribute bestimmte konzeptuelle Aspekte des modellierten (Frame-)Konzepts repräsentieren, das durch den ,zentralen' Knoten, von dem die Attribut-Pfeile ausge-

$18 \mathrm{Zu}$ den formalen Aspekten dieses Ansatzes siehe Petersen (2007). Zu den Grundannahmen und programmatischen Positionen siehe Löbner (2015). 
hen, repräsentiert wird. Die Pfeil-Richtung gibt an, welcher Knoten dem jeweiligen Attribut als Wert zugewiesen ist. Da von jedem Knoten-Punkt wiederum Attribut-Pfeile ausgehen können, denen wiederum Wert-Knoten zugewiesen sind, ergibt sich so eine rekursive Modellierungsstruktur, die es erlaubt, KonzeptStrukturen bis in feinste Verästelungen zu beschreiben. Im Fall des abgebildeten ,Tatobjekt'-Teilframes wäre es bspw. möglich, die Modellierung am ,Sache'-Knoten fortzusetzen, indem dort Attribute angedockt werden, die bestimmte Aspekte des Rechtsbegriffs ,Sache“ repräsentieren, und denen jeweils wiederum bestimmte Werte zugeordnet sind. Insgesamt ergibt sich so eine Modellierung in der Form funktionaler Beschreibungen, die bspw. so zu lesen sind: Die Gegenstandsart [= Attribut] eines (Wegnahme-)Tatobjekts [= zentraler Knoten des ,Tatobjekt'-Teilframes] ist: Sache [= Wert].

Der Teil-Frame modelliert die Bedingungen, die ein Objekt erfüllen muss, um Tatobjekt einer Wegnahme-Handlung im Sinne von $\S 242$ StGB sein zu können. Tatobjekt eines Diebstahl-Delikts kann demnach nur etwas sein, das die folgenden Bedingungen erfüllt: Es ist (im rechtlichen Sinne) eine Sache, es ist eigentumsfähig und es ist mobil - d. h. ihm kommt die im Normtext so genannte Eigenschaft zu, „beweglich“ $\mathrm{zu}$ sein. ${ }^{19}$ Unmittelbar einleuchtend ist sicherlich das Kriterium der „Beweglichkeit“ (Mobilität): Immobilien sind zwar Sachen im Sinne des Sachenrechts und eigentumsfähig, können jedoch nicht wie Uhren, Handtaschen etc. ,weggenommen' werden. Etwas komplexer verhält es sich mit den zwei anderen Kriterien: Zum einen kann es der Fall sein, dass etwas nicht eigentumsfähig ist, weil es keine Sache im rechtlichen Sinne ist, etwa wenn es sich um ein Immaterialgut handelt (eine Idee, eine Erfindung oder ein geistiges Werk), an dem man nicht Eigentümer, sondern ,Rechteinhaber' ist und dessen ,Diebstahl' darum nicht unter $§ 242$ StGB verhandelt wird (vgl. LK § 242, Rn 9). Zum anderen kann

${ }^{19}$ Das im Normtext genannte Merkmal „fremd“ ist in der Modellierung lediglich implizit, über das Merkmal der Eigentumsfähigkeit, enthalten. „Fremd“ impliziert, dass das Tatobjekt nicht zum Allein-Eigentum des Täters gehört - weswegen vorausgesetzt sein muss, dass das Tatobjekt eigentumsfähig ist. Personen sind daher als Tatobjekte ausgeschlossen, da sie aufgrund ihrer Eigenschaft als Rechtssubjekte nicht eigentumsfähig sind (vgl. MüKo § 90 BGB, Rn 2). Tiere sind zwar laut $§ 90 \mathrm{a}$ BGB keine Sachen, werden im strafrechtlichen Sinne jedoch dazugezählt. Vgl. LK § 242 StGB, Rn 8: „Nichtmenschliche Lebewesen, insbesondere Pflanzen, Pilze, Tiere, sind Sachen im strafrechtlichen Sinne.“ Pflanzen und Tiere sind als Handelsware eigentums- und darum „verkehrsfähig“, woraus sich dann die Möglichkeit zum Vieh-, Feld- und Forstdiebstahl ergibt, die zum „historischen Kern des Diebstahlrechts“ zählen (LK, ebd.). 
etwas zwar eine Sache sein, ist aber u. U. nicht eigentumsfähig, weil ihm die ,Verkehrsfähigkeit', d. h. die Möglichkeit, damit (legal) Handel zu treiben, abgesprochen wird. Dies ist etwa der Fall bei Drogen oder Waffen, wenn sie „mehr oder weniger weitgehenden Umgangsverboten unterliegen“ (LK § 242, Rn 31), sodass deren Besitz u. U. schon aufgrund der bestehenden Umgangsverbote strafrechtlich sanktionierbar ist - unabhängig davon, wie der Besitz an diesen Sachen ,erworben' wurde.

Was den Tatobjekt-Frame, in dem alle diese Aspekte modelliert sind, auszeichnet, ist aber nicht nur, dass er sich von der Ebene der Instantiierung löst, sondern auch, dass seine Struktur-Elemente nicht mehr unmittelbar auf die WegnahmeSituation bezogen sind, sondern stattdessen die allgemeine konzeptuelle Struktur eines im Wegnahme-Geschehen involvierten Partizipanten repräsentieren. Mit anderen Worten: Der Tatobjekt-Frame modelliert kein Verbal-Geschehen, das sich durch einen Satz repräsentieren ließe, der das mit dem Geschehen assoziierte Verb enthält, sondern er modelliert ein nominales Konzept. Das auf das TatobjektKonzept angewendete Frame-Format mit seinen Strukturelementen ,Attribut" und ,Wert' eignet sich zwar besonders gut für nominale Konzepte, ist aber durchaus auch auf verbale Konzepte übertragbar. So lassen sich auch die mit dem Verb wegnehmen assoziierten Partizipanten als ,attributive' Aspekte des WegnahmeKonzepts auffassen, deren jeweilige ,Wegnahme'-spezifischen Rollen über ihre entsprechenden Wert-Zuweisungen ausgedrückt werden können. ${ }^{20}$ Auf diese Weise lassen sich dem Wegnahme-Konzept (repräsentiert durch einen ,zentralen“

${ }^{20}$ Darum ,erben' die in den Knoten angegebenen Werte häufig konzeptuelle Aspekte des Attributs, dem sie zugeordnet sind. So bedeutet bspw. Täterschaft immer auch, Agens der Tathandlung zu sein. Dies ist ein Punkt, den auch Barsalou (1992) hervorhebt, an dessen Frame-Konzept sich das Düsseldorfer Modell in vielen Punkten anlehnt. Attribute sind laut Barsalou Konzepte, die bestimmte Aspekte einer Kategorie benennen: „Color becomes an attribute when viewed as an aspect of bird, and location becomes an attribute when viewed as an aspect of vacation (1992, $30 \mathrm{f}$.). Werte sind „subordinate concepts of an attribute. Because values are subordinate concepts, they inherit information from their respective attribute concepts" $(1992,31)$. Dies ist sicherlich eine gute Charakterisierung von Attributen und Werten von Gattungskonzepten (vgl. bspw. Barsalous carFrame; 1992, 30), trifft aber vielleicht weniger auf Attribute und Werte von event-Konzepten wie etwa Barsalous vacation-Frame zu (vgl. ebd., 38). Des Weiteren wird dadurch, dass Barsalou auch Attributen und Werten Konzept-Status zuspricht, der Rekursivitätscharakter seines Frame-Modells noch einmal potenziert. Denn so lassen sich auch Wert- und Attribut-Konzepte als Frames auffassen, denen selbst wieder Attribute und Werte zugeordnet werden können. (Im Düsseldorfer Modell ließe sich diese Ebene in Bezug auf die Attribute jedoch nicht abbilden, da die Attribute - anders als in Barsalous Modell - nicht als Knoten repräsentiert werden.) 
Wegnahme-Knoten) zunächst drei Attribut-Aspekte zuweisen: Agens, Patiens und Objekt (repräsentiert durch die jeweiligen Attribut-Pfeile), denen wiederum jeweils ein spezifischer Wert zugewiesen ist: Täter, Geschädigter und Tatobjekt (repräsentiert durch die jeweiligen Wert-Knoten). Auch diese Modellierung ist als eine Liste funktionaler Beschreibungen lesbar:

(i) Agens des Wegnahme-Geschehens ist der Täter.

(ii) Patiens des Wegnahme-Geschehens ist der Geschädigte.

(iii) Objekt des Wegnahme-Geschehens ist das Tatobjekt.

Diese drei Aspekte können dann den Ausgangspunkt für eine vorläufige - und natürlich noch unzureichende - Frame-Modellierung des Wegnahme-Konzepts bilden, der auf dem Wortlaut des Normtextes beruht (Abb. 4).

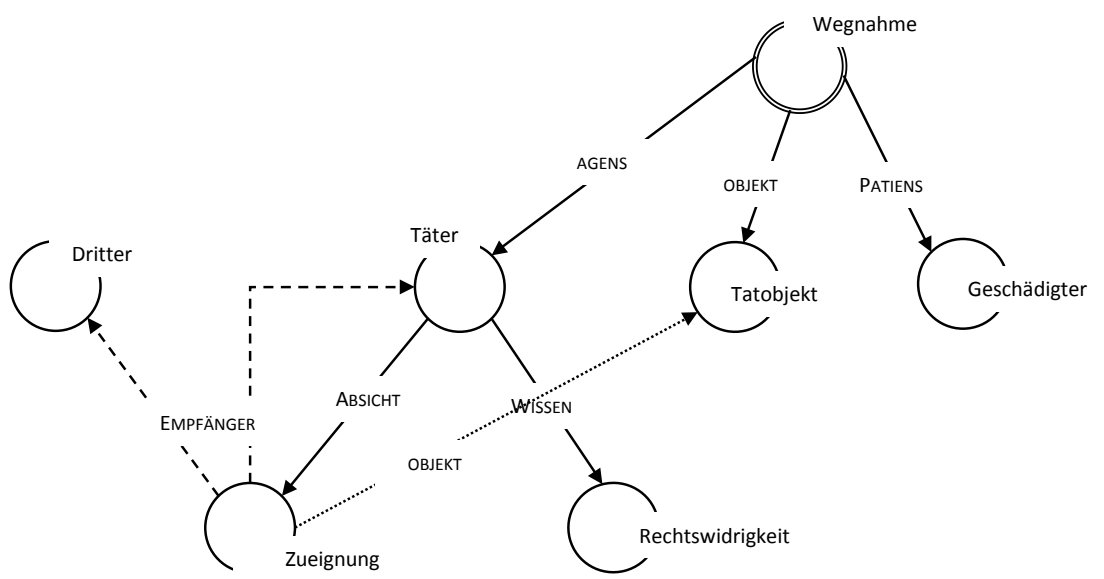

Abb. 4: ,Einfacher` Diebstahl-Frame, angelehnt an den Wortlaut des $§ 242$ Abs. 1 StGB

Entsprechend dem Wortlaut in $§ 242$ StGB Abs. 1 modelliert der Frame in Abb. 4 Diebstahl als Wegnahme einer fremden beweglichen Sache, in der Absicht, sie sich oder einem Dritten rechtswidrig zuzueignen. ${ }^{21}$ Neben den oben aufgelisteten

${ }^{21}$ Vgl. noch einmal Abs. 1: „Wer [Täter] eine fremde bewegliche Sache [Tatobjekt] einem anderen [Geschädigter] in der Absicht wegnimmt, die Sache sich oder einem Dritten rechtswidrig zuzueignen, wird mit Freiheitsstrafe bis zu fünf Jahren oder mit Geldstrafe bestraft.“ 
Partizipanten-Aspekten (Täter, Geschädigter, Tatobjekt) modelliert der Frame noch die weiteren in Abs. 1 genannten Aspekte: das Wissen des Täters um die Rechtswidrigkeit, die täterseitige Absicht der Zueignung, sowie, dass der ,Empfänger' der (beabsichtigten) Zueignung der Täter selbst oder ein Dritter sein kann. (Dass der Täter die Sache u. U. nicht sich selbst, sondern einem Dritten zueignen möchte, wird durch die gestrichelten Attributpfeile indiziert.) Und schließlich ist im Frame noch modelliert, dass das Tatobjekt nicht nur Objekt der Wegnahme, sondern natürlich auch Objekt der (beabsichtigten) Zueignung ist.

Vergleicht man die Modellierung in Abb. 4 mit der in FrameNet vorgeschlagenen Konzeptualisierung, so fällt auf, dass der Normtext das SOURCE-Element, das laut FrameNet zu den „core elements“ zählt, überhaupt nicht berücksichtigt und stattdessen einen weiteren Partizipanten ins Spiel bringt, der in der FrameNetKonzeptualisierung fehlt: den Empfänger der Zueignung, der, wie in Abs. 1 explizit herausgestellt wird, nicht mit dem Täter identisch sein muss.

Dieser lässt sich zwar ohne Probleme durch ein Dativ-Argument realisieren, aber ohne vorausgesetztes Situationswissen bleibt hierbei völlig offen, ob im Dativ-Argument der Empfänger oder der Geschädigte realisiert ist:

(2) Arno stielt Ute eine Perlenkette. ${ }^{22}$

Unabhängig von der Frage der ausdrucksseitigen Realisierbarkeit der involvierten Partizipanten (und ihrer Zuweisung auf die entsprechenden Argument-Konstituenten) erfassen solche Analysen jedoch weder die für das juristische Diebstahl-Konzept so zentrale Unterscheidung von Wegnahme und Zueignung noch tragen sie dazu bei, die Konsequenzen zu erhellen, die sich daraus für das Dieb-

${ }^{22}$ Auch die Realisierung aller denkbaren Partizipanten (Täter, Geschädigter, Tatobjekt, Empfänger) ist möglich, setzt bzgl. der Rollen-Zuweisung aber ebenfalls eine Menge Vorwissen (bzw. Situationswissen) voraus: Arno stiehlt Ute Giselas Perlenkette. Hier ist für das Dativ-Argument auch die Empfänger-Lesart möglich (in welchem Fall alle vier Partizipanten-Rollen besetzt wären). In der anderen Lesart (die vielleicht salienter ist) ist Ute diejenige, der die Kette weggenommen wird, sodass Arno, wie im prototypischen Fall, Täter und Empfänger der Zueignung in einer Person ist. Der Umstand, dass nicht Ute, sondern Gisela Eigentümerin der Kette ist, spielt für die strafrechtliche Bewertung der Tat Arnos keine Rolle. Möglich wäre auch, dass Ute die Kette schon von Gisela gestohlen hat und Arno sie im Anschluss Ute wegnimmt. Auch dies würde für die strafrechtliche Bewertung der Tat keine Rolle spielen. Lediglich in der folgenden, als Entscheidungsfrage realisierten Aufforderung lassen sich die jeweiligen Partizipanten-Rollen durch ihre deiktische Situierung sicher zuweisen: (Ute zu Arno) Stiehlst du mir Giselas Perlenkette? 
stahl-Konzept ergeben. Ohnehin ist es so, dass eine framesemantische Modellierung des Rechtskonzepts ,Diebstahl' nicht bei der Benennung der darin involvierten Partizipanten stehen bleiben kann. Was in der Frame-Modellierung in Abb. 4 (und ebenso in der FrameNet-Konzeptualisierung) völlig unbestimmt bleibt, ist die Modellierung des Wegnahme-Begriffs selbst, der in den Kommentaren über die Konzepte ,Bruch` und ,Begründung' expliziert wird.

\section{Bruch und Begründung framesemantisch}

Ein weiterer zentraler Aspekt des Diebstahl-Konzepts ist der mit der WegnahmeHandlung notwendig einhergehende Umstand, dass die Sache von einer Person auf die andere übergeht: Die Wegnahme bewirkt, dass jemand, der eine Sache zuvor in Gewahrsam hatte, sie jetzt nicht mehr in Gewahrsam hat, und an seiner Stelle nun der Wegnehmende Inhaber des Gewahrsams an der Sache ist. Dieser zunächst trivial erscheinende Sachverhalt wird in der Kommentarliteratur unter dem Stichwort der „Gewahrsamsverschiebung“ verhandelt. Stellvertretend für viele andere, mehr oder weniger gleichlautende Kommentarstellen sei hier eine einschlägige Stelle aus dem Münchner Kommentar angeführt:

Wegnahme setzt die Verschiebung des Gewahrsams voraus. Daher muss er auf Seiten des alten Gewahrsamsinhabers durch die Tathandlung aufgehoben und anschließend neuer Gewahrsam begründet werden. Mit der Begründung neuen Gewahrsams ist die Tat vollendet. (MüKo § 242 StGB, Rn 71)

Die Kommentarliteratur deutet Wegnahme darum als Handlung, die sich aus zwei Handlungsteilen zusammensetzt: „Erster Handlungsteil ist der Bruch fremden Gewahrsams“, der sich dadurch auszeichnet, dass die „tatsächliche[] Sachherrschaft [...] des bisherigen Gewahrsamsinhabers ohne dessen Willen aufgehoben ist“ (Schönke/Schröder § 242 StGB, Rn 35). „Als zweiter Handlungsteil ist zwecks Vollendung der Wegnahme die Begründung neuen Gewahrsams auf Täterseite erforderlich“ (ebd., Rn 37). Gewahrsam gilt als begründet, wenn der Täter „die Herrschaft über die Sache derart erlangt hat, dass er sie ohne Behinderung durch den alten Gewahrsamsinhaber ausüben und dieser seinerseits ohne Beseitigung der Verfügungsgewalt des Täters nicht mehr über die Sache verfügen kann“ (ebd., Rn 38). Wann bzw. ob dies schon der Fall ist, lässt sich nur auf der Basis der jeweiligen Einzelfall-Bedingungen beurteilen (MüKo § 242 StGB, 
Rn $71 \mathrm{ff}$.). ${ }^{23}$ Wer beispielsweise in einem Selbstbedienungsladen eine Sache ergreift und heimlich einsteckt, und dies unmittelbar danach vom Personal entdeckt wird, hat Gewahrsam schon begründet, obwohl er die Sache noch nicht fortschaffen konnte (Schönke/Schröder § 242 StGB, Rn 39). Dass der Täter die Sache einstecken konnte, genügt, um von ,Sachherrschaft' sprechen zu können (LK § 242 StGB, Rn 96). Anders sieht es aus, wenn die Sache zu groß ist, um ,einfach so“ eingesteckt und fortgeschafft werden zu können. In solchen Konstellationen lässt sich von Sachherrschaft oft erst dann sprechen, wenn der Täter die Sache aus dem „räumlichen Machtbereich“ des rechtmäßigen Besitzers herausschaffen konnte (ebd., Rn 97).

Aus der Perspektive von Bruch und Begründung ist das Ergreifen und Einstecken einer Sache ohne Frage ein prototypischer Fall. In dieser Hinsicht weniger prototypisch ist sicherlich der schon oben erwähnte Fall des Schäfers, der seine Schafe vorsätzlich und unbefugt auf einem fremden Grundstück hat weiden lassen. In Bezug auf den Bruch-Aspekt wird der Tathergang im Urteil zunächst folgendermaßen gedeutet:

Der Bruch [des] Gewahrsams und die Begründung neuen Gewahrsams durch den Angekl. ist darin zu sehen, daß die Schafe sich Gras und Klee einverleibt haben. So hat das OLG Köln [...] entschieden, daß derjenige, der in einem Selbstbedienungsladen eine Flasche öffne und daraus trinke, den Gewahrsam an dem Getränk deshalb inne habe, weil in dem Augenblick des Trinkens der Inhaber des Ladens von dem Gewahrsam an dem im Mund des Täters befindlichen Teil des Getränks ausgeschlossen werde, da es in diesem Augenblick nach der Lebensauffassung eindeutig der Herrschaftssphäre des Täters zuzuordnen sei. [...] Übertragen auf den vorliegenden Fall bedeutet dies, daß die Herrschaftsmacht des bisherigen Gewahrsamsinhabers in dem Moment, in dem die Schafe das Gras vom Boden abgerissen hatten und im Maul hielten, aufgehoben war, da seine Einwirkungsmöglichkeiten auf das Gras damit nicht mehr bestanden. Daß der Angekl. den Gewahrsam nicht durch eine eigene Handlung gebrochen hat, sondern dieses durch die Tiere geschah, ist unerheblich, da ihm das Verhalten der Tiere zugerechnet wird [...]. (NStZ 1993, 543)

Die Gewahrsamsbegründung wird im Urteil - ganz im Einklang mit der Explikation der einschlägigen Kommentarliteratur - daraus abgeleitet, dass der Schäfer

${ }^{23}$ Hinzu kommt, dass die Frage, ob Gewahrsam schon begründet wurde oder noch nicht, ohnehin nur dann entscheidungsrelevant ist, wenn der Täter , auf frischer Tat betroffen' wird - und nicht mehr, wenn er die Sache schon längst fortgeschafft und gesichert hat (vgl. LK § 242 StGB, Rn 85). 
durch das Weidenlassen der Schafe „tatsächliche Herrschaftsgewalt über die Sache erlangt" hat:

Indem die Schafe das Gras vom Boden abrissen und im Maul hielten, hat der Angekl. es zwar nicht selbst ergriffen, da ihm jedoch die Schafe als Werkzeuge und damit quasi als ,verlängerter Arm' fungierten, ist ihm deren ,Inbesitznahme' über Gras und Klee zuzurechnen. (NStZ 1993, 544)

Ob nun also der Gewahrsamswechsel durch Ergreifen und Einstecken der Sache herbeigeführt wurde oder durch Schafe, die als „Werkzeuge“ und „verlängerter Arm" des Schäfers gewirkt haben - ausschlaggebend für die Subsumtion unter Bruch und Begründung ist, dass durch den Tathergang bestimmte Effekte erzielt wurden, die sich für beide Handlungsteile allgemein benennen lassen: Bruch bewirkt, dass der Geschädigte seinen Gewahrsam an der Sache verliert, Begründung bewirkt, dass der Täter Sachherrschaft über die Sache erlangt.

Zwar ist es im prototypischen Fall so, dass sich die Begründung unmittelbar aus der Bruchhandlung ergibt. Aber Bruch und Begründung stehen nicht notwendig in einem „engen zeitlichen und räumlichen Zusammenhang“, beispielsweise dann nicht, wenn der Täter Pakete von einem Güterzug abwirft, um sie später vom Bahndamm abzuholen (vgl. MüKo § 242, Rn 71). Es sind solche oder ähnliche Fall-Konstellationen, die zeigen, dass sich Gewahrsamsbegründung nicht einfach nur als Effekt von Bruchhandlungen konzeptualisieren lässt. Im Fall des Ladendiebstahls ist dies zwar insofern der Fall, als durch das Ergreifen und Einstecken der Ware auch die Begründung unmittelbar erfolgt ist, ebenso wie sich im Fall der unbefugt weidenden Schafe die Begründung unmittelbar aus dem Abfressen des Grases ergibt. Aber dennoch sind Fall-Konstellationen möglich siehe das Güterzug-Beispiel -, in denen sich für beide Wegnahme-Aspekte spezifische Handlungen angeben lassen, durch die die jeweiligen bruch- und begründungsspezifischen Effekte erzielt werden. So ist das Abwerfen der Pakete vom Güterzug zwar Bruch, weil der Geschädigte dadurch seinen Gewahrsam an den Paketen verliert, aber hieraus folgt noch keine Begründung, weil die Pakete durch das Abwerfen zunächst gewahrsamslos werden. Erst das Abholen der gewahrsamslosen Pakete vom Bahndamm ist die Begründungshandlung, die bewirkt, dass der Täter nun auch Sachherrschaft über sie erlangt hat.

Da sich Bruch und Begründung vor diesem Hintergrund nicht nur als Handlungsteile, sondern durchaus auch als Teil-Handlungen begreifen lassen, bietet es 
sich an, beide Wegnahme-Aspekte als Teil-Frames zu modellieren. Dies ist besonders naheliegend für den Bruch-Aspekt, denn dort ist es aufgrund der in bestimmten Fall-Konstellationen u. U. klärungsbedürftigen Frage, ob von versuchtem oder vollendetem Diebstahl auszugehen ist, prinzipiell immer möglich, Bruch als individualisierbare Teil-Handlung ohne jegliche Begründungsaspekte zu konzeptualisieren. ${ }^{24}$

In Anlehnung an den vorläufigen Wegnahme-Frame in Abb. 4 bietet es sich daher an, das Bruch-Konzept zunächst über die involvierten Partizipanten (Täter, Tatobjekt, Geschädigter) sowie des Weiteren über den Effekt zu modellieren, der in den Kommentaren einhellig als Gewahrsamsverlust charakterisiert wird (siehe Abb. 5).

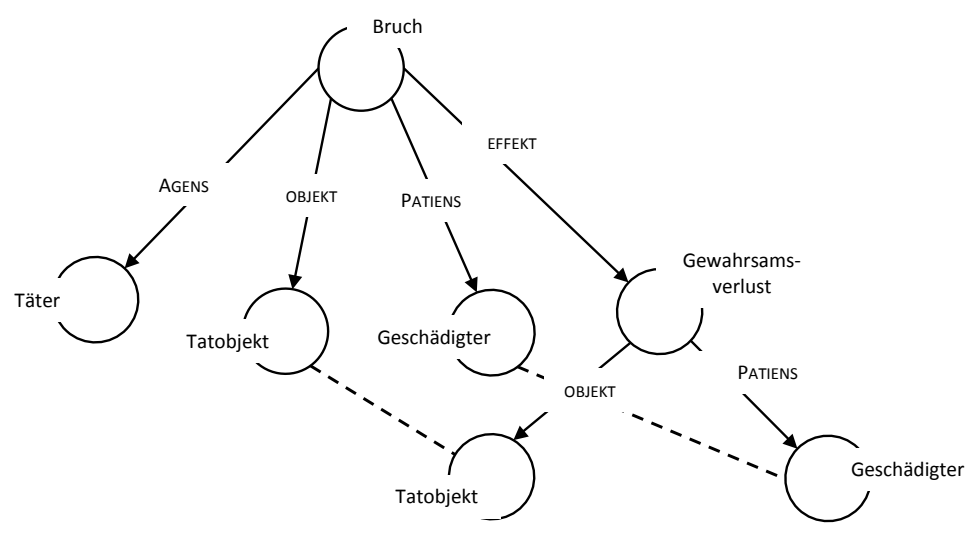

Abb. 5: Modellierung von Bruch als Bewirkens-Relation mit Aktanten

Auf diese Weise ergeben sich zunächst wieder funktionale Beschreibungen, die, ausgehend vom (zentralen) Bruch-Knoten, folgendermaßen zu lesen sind:

(i) Agens der Bruch-Handlung ist der Täter;

(ii) Objekt der Bruch-Handlung ist das Tatobjekt;

(iii) Patiens der Bruch-Handlung ist der Geschädigte;

(iv) Effekt des Bruch-Handlung ist der Gewahrsamsverlust.

${ }^{24}$ Für mögliche Fall-Konstellationen, in denen nur Bruch vorliegt, vgl. LK § 242 StGB, Rn 89 f. 
Zur Vervollständigung des Gesamtbildes ist dann noch zu ergänzen, wer Gewahrsamsverlust erleidet, sowie schließlich, woran Gewahrsamsverlust besteht:

(v) Patiens des Gewahrsamsverlusts ist der Geschädigte;

(vi) Objekt des Gewahrsamsverlusts ist das Tatobjekt.

Die Frage ist nun, wie sich die Angaben in (v) und (vi) in die Modellierung einbauen lassen. Bindet man sie, so wie in Abb. 5, an den Gewahrsamsverlust-Knoten an, kommt es in Bezug auf das Tatobjekt und den Geschädigten zu unerwünschten Doppelungen (in der Modellierung hervorgehoben durch die gestrichelten Linien). Die Werte für das Objekt des Gewahrsamsverlusts (das Tatobjekt) sowie für den ,Betroffenen“ (d. h. das Patiens) des Gewahrsamsverlusts (der Geschädigte) sind identisch mit den Werten der direkt am Bruch-Knoten anschließenden Attribute. Diese Identität ist jedoch nicht nur konzeptueller, sondern auch ,realweltlicher ' Natur, denn das Tatobjekt ist nicht nur das Objekt, an dem (seitens des Geschädigten) Gewahrsamsverlust besteht, sondern natürlich auch das Objekt, um dessentwillen der Täter den Bruch begeht. Und der Geschädigte ist sowohl derjenige, der vom Gewahrsamsverlust (am Tatobjekt) betroffen ist, als auch derjenige, der ,Betroffener ' des Bruch-Geschehens ist. Diese Doppelungen lassen sich auflösen, indem man zunächst das Patiens-Attribut am BruchKnoten streicht, sodass der Geschädigte im Frame nur einmal, als Wert des vom Gewahrsamsverlust-Knoten ausgehenden Patiens-Attributs, realisiert wird. Und die Doppelung in Bezug auf das Tatobjekt lässt sich eliminieren, indem der Tatobjekt-Knoten sowohl Wert des Bruch-Attributs ,Objekt' als auch Wert des Gewahrsamsverlust-Attributs ,Objekt' ist.

In der revidierten Modellierung (siehe Abb. 6) sind die unerwünschten Doppelungen beseitigt. Dennoch erfasst der Frame alle Relationen, die zwischen Tatobjekt und Bruch sowie zwischen Tatobjekt und Gewahrsamsverlust bestehen. Effekt des Bruchs ist der Gewahrsamsverlust, welcher sowohl auf den Geschädigten als auch auf das Tatobjekt bezogen ist: Der Geschädigte erleidet durch den Bruch Gewahrsamsverlust an dem Tatobjekt. Das Tatobjekt ist sowohl das Objekt, an dem Gewahrsam verloren gegangen ist, als auch das Objekt des Bruchs. Diese Identität wird im Frame dadurch ausgedrückt, dass der Tatobjekt-Knoten Wert zweier Attribute ist: sowohl des Objekt-Attributs, das vom Bruch-Knoten ausgeht, als auch des Objekt-Attributs, das zum Gewahrsamsverlust-Knoten gehört. 
Die Doppelung in Bezug auf den Geschädigten ist dadurch aufgehoben, dass der Geschädigte nicht als Partizipant des Bruchgeschehens konzeptualisiert wird, sondern als Patiens des Gewahrsamsverlusts.

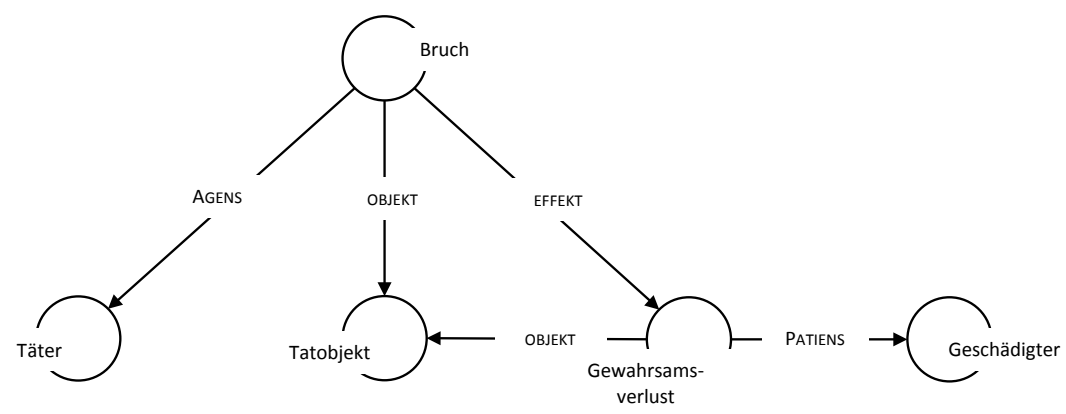

Abb. 6: Revidierte Bruch-Modellierung

Es ist wichtig, sich darüber im Klaren zu sein, dass sich diese Modellierung in weiten Teilen von valenzgrammatisch inspirierten Konzeptualisierungen löst, in denen die vom zentralen Knoten abgehenden Attribut-Wert-Paare als Argumente des Verbs gedeutet werden und die Wert-Knoten die den Argumenten jeweils zugewiesenen semantischen Rollen repräsentieren. Zwar lassen sich alle Beteiligte (Täter, Geschädigter und Tatobjekt) in einem Satz, der ein Bruch-Geschehen repräsentiert, realisieren - so wie etwa in (3):

(3) Arno [Täter] bricht Utes [Geschädigter] Gewahrsam an ihrer Handtasche [Tatobjekt].

Aber diese Realisierungen finden sich nicht in analoger Weise in der Argumentstruktur des Satzes wieder, der lediglich aus dem transitiven Verb (bricht), dem Subjekt-Argument (das den Täter repräsentiert) und dem Objekt-Argument besteht, wobei im Kopf der Objektkonstituente nicht einer der Partizipanten realisiert ist, sondern das Substantiv ,Gewahrsam', dem die zwei verbliebenen Partizipanten lediglich durch Attribut-Konstituenten zugewiesen sind. Insofern findet sich in der Argumentstruktur des Satzes keine ,Abbildung' der Struktur des Realgeschehens wieder - so wie es bspw. für Fälle angenommen wird, in denen das 
Objekt des transitiven Satzes auch das Patiens des vom Agens ausgeführten Verbalgeschehens repräsentiert, wie etwa in: Arno zerbricht eine Vase. Die fehlende Strukturhomologie zwischen Realgeschehen und Satz in (3) erklärt sich zum Teil auch dadurch, dass es im Grunde verfehlt ist, den Satz als Repräsentation eines Realgeschehens auffassen zu wollen. Der Satz drückt vielmehr die Subsumtion eines Realgeschehens (z. B. das Ergreifen einer Handtasche) unter den Rechtsbegriff des Gewahrsamsbruchs aus. Im Hinblick auf das tatsächliche Geschehen ,vollständiger' wäre daher die Variante in (4):

(4) Arno bricht Utes Gewahrsam an ihrer Handtasche, indem er die Handtasche ergreift.

Was im Hauptsatz ausdrucksseitig in finites Verb (bricht) und Kopf der ObjektKonstituente (Gewahrsam) zerfällt, ist das begriffliche Konzept ,Gewahrsamsbruch', unter das das Realgeschehen (das Ergreifen der Handtasche) subsumiert ist. In der Frame-Modellierung abstrahiert der Bruch-Knoten von den tatsächlichen Details des Realgeschehens (etwa ob der Bruch durch Ergreifen oder auf irgendeine andere Weise vollzogen wird), weshalb die Knotenbezeichnung „Bruch`als ,Label` für das Rechtskonzept ,Gewahrsamsbruch` fungiert - das es ja im Frame zu modellieren galt.

Auf nahezu analoge Weise lässt sich das Begründungskonzept modellieren (Abb. 7).

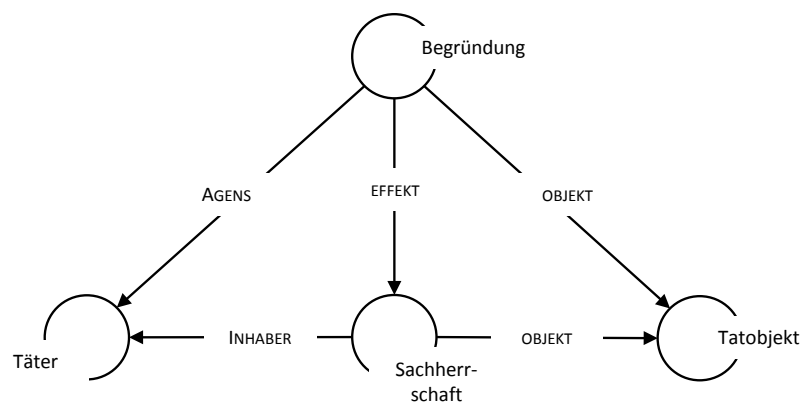

Abb. 7: Teilframe Begründung 
Die mit der Begründung assoziierten Partizipanten sind Agens (Täter) und Objekt (Tatobjekt), die als Attribut-Werte-Paar an den zentralen Knoten angehängt sind. Ebenso wie im Bruch-Frame ist auch dort ein Effekt-Attribut an den zentralen Knoten angebunden. Effekt der Begründung ist der neu etablierte Gewahrsam, beziehungsweise hier: die neu etablierte (täterseitige) Sachherrschaft. Die Sachherrschaft steht in Relation zu ihrem Inhaber (dem Täter) und zu ihrem Objekt (dem Tatobjekt). Das Objekt der Sachherrschaft ist auch das Objekt der Gewahrsamsbegründung, wodurch ausgedrückt wird, dass der Täter Inhaber der Sachherrschaft über das Tatobjekt ist.

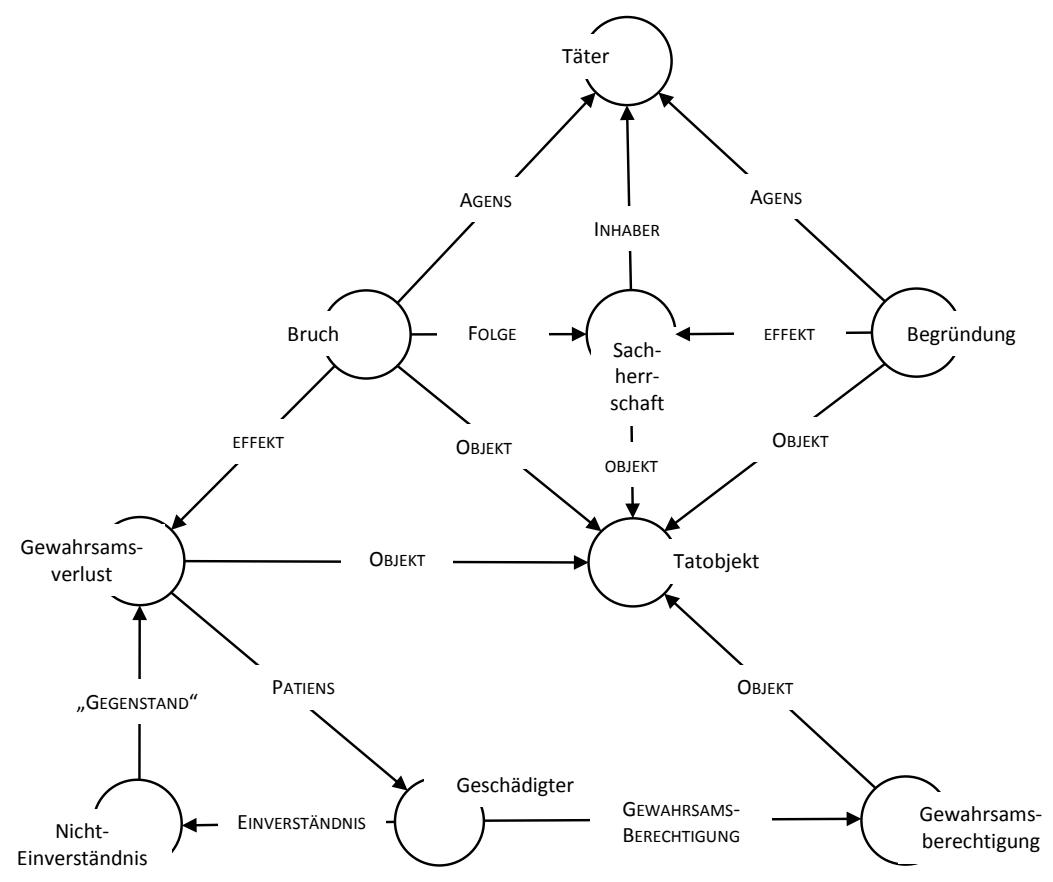

Abb. 8: Bruch fremden und Begründung neuen Gewahrsams

Fügt man beide Handlungsteile (Bruch und Begründung) zusammen, ergibt sich die Modellierung in Abb. 8, die darstellungstechnisch bedingt allerdings nicht nur 
ein vollkommen anderes Arrangement der Wert-Knoten und Attribut-Pfeile aufweist, sondern auch Amalgamierungen in Bezug auf den Täter und das Tatobjekt enthält: Der Täter ist sowohl Agens des Bruchs als auch Agens der Begründung und das Tatobjekt ist Objekt des Bruchs wie auch Objekt der Begründung.

Des Weiteren modelliert der Frame noch den Aspekt des Nicht-Einverständnisses des Geschädigten in seinen Gewahrsamsverlust sowie den Umstand, dass der Geschädigte berechtigt ist, Gewahrsam am Tatobjekt zu haben. Anders als die anderen Wert-Zuschreibungen (Agens des Bruchs ist der Täter, Objekt des Bruchs ist das Tatobjekt etc.) fügen sich diese Aspekte allerdings nicht so ,glatt ${ }^{\star}$ in das funktionale Beschreibungsformat ein. In Bezug auf den EinverständnisAspekt ist die Modellierung folgendermaßen zu verstehen: Laut Kommentarliteratur ist der Einverständnis-Aspekt „tatbestandsausschließendes Merkmal“: Bruch liegt nur dann vor, wenn der Gewahrsamsverlust „gegen oder ohne den Willen des Berechtigten erfolgt, es also am so genannten tatbestandsausschließenden Einverständnis fehlt“ (Joecks $§ 242$ StGB, Rn 10). In Bezug auf den Einverständnis-Aspekt sind somit zwei ,Zustände“ denkbar: Bzgl. des Gewahrsamsverlusts besteht seitens des Gewahrsamsberechtigten Einverständnis oder es besteht kein Einverständnis - in welchem Fall Bruch vorliegt. Der auf das Einverständnis-Attribut bezogene Wert ist darum ,Nicht-Einverständnis‘, welches einen ,Gegenstand' hat, nämlich den Gewahrsamsverlust (über das Tatobjekt).

Die Modellierung gibt also den folgenden Sachverhalt wieder: Der Geschädigte zeigt kein erkennbares Einverständnis (bzw. hat kein Einverständnis zu erkennen gegeben) bzgl. seines Gewahrsamsverlusts an dem Tatobjekt, für das er Gewahrsamsberechtigung hat. Die Gewahrsamsberechtigung ist der zweite Aspekt, der auf den Geschädigten bezogen ist und der die Eigentums- bzw. Besitz-Relation zwischen Geschädigtem und Tatobjekt benennt: Der Geschädigte hat Gewahrsamsberechtigung an dem Tatobjekt. ${ }^{25}$

25 Man könnte hier einwenden, dass sich dieser Aspekt funktional doch wesentlich ,glatter ‘ beschreiben ließe: Inhaber der Gewahrsamsberechtigung ist der Geschädigte. Allerdings würde diese Modellierung die Attribuierungsperspektive umkehren (die Gewahrsamsberechtigung hat einen Inhaber: den Geschädigten) - was darüber hinaus auf der Seite des Komplementär-Aspekts (der NichtBerechtigung des Täters am Gewahrsam über das Tatobjekt, siehe Abb. 9: vollständiger DiebstahlFrame) keine so ,glatte ' Modellierung ergibt: Inhaber der Gewahrsamsnichtberechtigung ist der Täter. Die Komplementarität dieser zwei Aspekte bringt zum Ausdruck, was aus dem fehlenden Einverständnis in den Gewahrsamsübergang notwendig hervorgeht: dass der Gewahrsam des Geschädigten am Tatobjekt rechtmäßig ist, der Gewahrsam des Täters am Tatobjekt jedoch nicht. 
Ein weiterer im Frame modellierter Aspekt ist die Relation zwischen Bruchhandlung und täterseitiger Sachherrschaft, die als Folge-Beziehung charakterisiert ist: Folge der Bruchhandlung (deren Agens der Täter ist) ist die (täterseitige) Sachherrschaft. Dies wirft wiederum die Frage auf, ob es nicht angemessener wäre, Begründung lediglich als Folge-Aspekt der Bruchhandlung zu modellieren, wodurch ihr dann allerdings der Status als eigenständiger, isolierbarer Handlungsteil abhandenkäme. Dies widerspräche jedoch der Motivation, die dieser Unterscheidung zugrunde liegt, und die darin besteht, dass Bruch und Begründung als parallele und gleichgewichtige Handlungsteile $\mathrm{zu}$ verstehen sind, mit deren Hilfe sich bestimmte begriffliche und rechtsprechungspraktische Fragen beantworten lassen, die sich aus dem Diebstahl-Konzept ergeben: etwa die Frage, nach welchen Kriterien zwischen Diebstahl und versuchtem Diebstahl unterschieden werden kann, sowie, damit zusammenhängend, ab wann und unter welchen Bedingungen Diebstahl als vollendet gilt. Insofern implizieren Bruch und Begründung also ein Nacheinander - wenn auch in der Regel eher logisch als zeitlich und nicht ein ,Enthaltensein' in dem Sinne, dass Gewahrsamsbegründung als Teilaspekt der Bruchhandlung aufzufassen wäre.

Was das vom Bruch-Knoten ausgehende Folge-Attribut zum Ausdruck bringt, ist das besondere Verhältnis zwischen Bruchhandlung und täterseitiger Sachherrschaft: Wer im Rahmen einer Wegnahme-Handlung im Sinne von § 242 StGB über ein Tatobjekt Sachherrschaft erlangt hat, muss notwendigerweise Bruch begangen haben. ${ }^{26}$ Andererseits bedeutet Bruch jedoch nicht, dass der Täter mit Notwendigkeit auch Sachherrschaft über das Tatobjekt erlangt. Wäre dies der Fall, so könnte man weder theoretisch noch praktisch zwischen versuchtem und vollendetem Diebstahl unterscheiden. Anders sieht dies bei den Bruch und Begründung jeweils zugeordneten Effekt-Werten aus. Weder lässt sich von Bruch sprechen, wenn es zu keinem Gewahrsamsverlust gekommen ist, noch kann von

Darum ist es für die Modellierung angemessener, Gewahrsamsberechtigung bzw. Gewahrsamsnichtberechtigung als Aspekte aufzufassen, die dem Geschädigten bzw. dem Täter zugeschrieben werden (und nicht umgekehrt Gewahrsamsberechtigung und Gewahrsamsnichtberechtigung als ,Entitäten` zu konzeptualisieren, denen jeweils ein Geschädigter bzw. ein Täter als ,Inhaber` zugeordnet sind).

${ }^{26}$ Dies zeigt sich etwa auch daran, dass bei rechtswidriger Sachherrschaft, die auf anderem Wege als durch Bruch erlangt wurde, nicht von Diebstahl, sondern von Unterschlagung oder Betrug gesprochen werden müsste. 
Begründung die Rede sein, wenn der Täter keine Sachherrschaft über das Tatobjekt erlangt hat. Dass dort beide Werte mit Notwendigkeit gelten, der Wert ,Sachherrschaft' in Bezug auf den Bruch-Aspekt jedoch nur, wenn die Wegnahme voll-

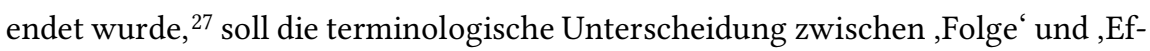
fekt ' indizieren (auch wenn sich dies in der Semantik dieser zwei Bezeichnungen natürlich nicht widerspiegelt).

\section{Der vollständige Wegnahme-Frame}

Für die vollständige Modellierung (siehe Abb. 9) sind jetzt noch die auf den Täter bezogenen „subjektiven“ Tatbestandsmerkmale zu ergänzen sowie der ,zentrale“ Wegnahme-Knoten, auf den die Teilaspekte ,Bruch` und ,Begründung' bezogen sind. Entsprechend der in der Kommentarliteratur üblichen Sichtweise, dass sich Wegnahme aus den „Handlungsteilen“ „Bruch“ und ,Begründung“ zusammensetzt, sind sie im Gesamt-Frame als die jeweiligen Werte zweier Attribute, die von einem zentralen Wegnahme-Knoten ausgehen, realisiert:

(i) Handlungsaspekt 1 der Wegnahme ist Bruch;

(ii) Handlungsaspekt 2 der Wegnahme ist Begründung.

Die subjektiven, auf den Täter bezogenen Tatbestandsmerkmale sind zunächst seine Absicht (Attribut) der Zueignung (Wert) sowie sein Wissen (Attribut) um ihre Rechtswidrigkeit (Wert). Darum ist die Zueignung Wert zweier Attribute: zum einen des auf den Täter bezogenen Absicht-Attributs, zum anderen des vom Rechtswidrigkeit-Knoten abgehenden ,Gegenstand'-Attributs: ,Gegenstand' des Wissens um die Rechtswidrigkeit ist die Zueignung. Empfänger (Attribut) der Zueignung ist entweder der Täter (Wert) oder ein Dritter (Wert). (Dass potenziell beide Empfänger sein können, wird durch die gestrichelten Attribut-Pfeile angezeigt.)

Erläuterungsbedürftig ist noch der Motivationsaspekt. Was die täterseitige Wegnahme motiviert, ist der Wille, über die Sache in einer „eigentümerähnlichen“"Weise zu verfügen (vgl. Fischer § 242 StGB, Rn 33). Auch für den Leipziger

\footnotetext{
${ }^{27}$ Nur aus der Perspektive des gesamten Wegnahme-Geschehens ist der auf den Bruch bezogene Wert ,Sachherrschaft' notwendig, nämlich insofern, als nur vollendete Wegnahme Gewahrsamsbegründung und damit täterseitige Sachherrschaft impliziert.
} 
Kommentar ist dies „Motiv oder Zweck des Diebstahls“: Durch die Wegnahme „kann sich der Dieb [...] an die Stelle des Eigentümers setzen und diesen damit [...] aus seiner Eigentümerposition verdrängen“ (LK §242 StGB, Rn 133). Der Wille, „wie ein Eigentümer über die Sache zu verfügen“ und damit auf Dauer Sachherrschaft über sie zu haben (Fischer, § 242 StGB, Rn 33 f.), erschöpft sich jedoch nicht in der bloßen Absicht, dies zu tun. Vielmehr besteht er solange fort, bis er aktiv aufgegeben wird. Ohne einen solchen Willen - der im Zivilrecht Besitzwille genannt wird (vgl. Staudinger, Eckpfeiler des Zivilrechts, V. Rn 8 f.) liegt Sachherrschaft nicht vor: „Da Sachherrschaft die Unterwerfung der Sache unter den eigenen Willen bedeutet, kann von ihr ohne einen solchen Willen nicht die Rede sein; will jemand eine Sache nicht beherrschen, hat er sie auch nicht in seiner tatsächlichen Gewalt“ (Staudinger, ebd. Rn 9). ${ }^{28}$ Was die Wegnahme somit täterseitig motiviert, ist, die Sache seinem Willen zu unterwerfen - wodurch dann erst die Voraussetzung dafür geschaffen ist, sie sich (oder einem Dritten) längerfristig zuzueignen (vgl. LK § 242, Rn 148 ff.).

Die Modellierung bringt auch diese weiteren, impliziten Relationen zwischen Täter und Tatobjekt zum Ausdruck, deren Beziehung sich somit nicht allein darin erschöpft, dass der Täter das Tatobjekt wegnimmt. Das Tatobjekt ist Objekt der Zueignung (die der Täter durch die Wegnahme beabsichtigt) sowie Objekt seines Eigentumswillens (d. h. das Objekt, um dessentwillen der Täter die Wegnahme ausführt).

${ }^{28}$ Dies bedeutet natürlich nicht, dass aus dem Besitzwillen notwendig Sachherrschaft folgt. Vielmehr ist es andersherum zu verstehen: Aus dem objektiven Sachverhalt, dass jemand (aktuell) Sachherrschaft über eine Sache innehat, lässt sich auf den subjektiven Sachverhalt schließen, dass er auch willens ist, diese auszuüben. Dennoch lässt sich beides getrennt denken: Jemand, dem eine Sache heimlich weggenommen wurde, vermeint lediglich, die Sachherrschaft innezuhaben. Dass aber sein Wille, die Sache zu besitzen, nach wie vor besteht, zeigt sich gerade daran, dass sie ihm ohne seinen Willen weggenommen wurde, dass er also gegen die Wegnahme in irgendeiner Form vorgegangen wäre, wenn er sie bemerkt hätte. Aus dieser Perspektive sind Besitzwille und (tatsächliche) Sachherrschaft also getrennt zu denken, denn andernfalls könnte weder von Gewahrsamsverlust „ohne Willen", noch von Bruch gesprochen werden. 


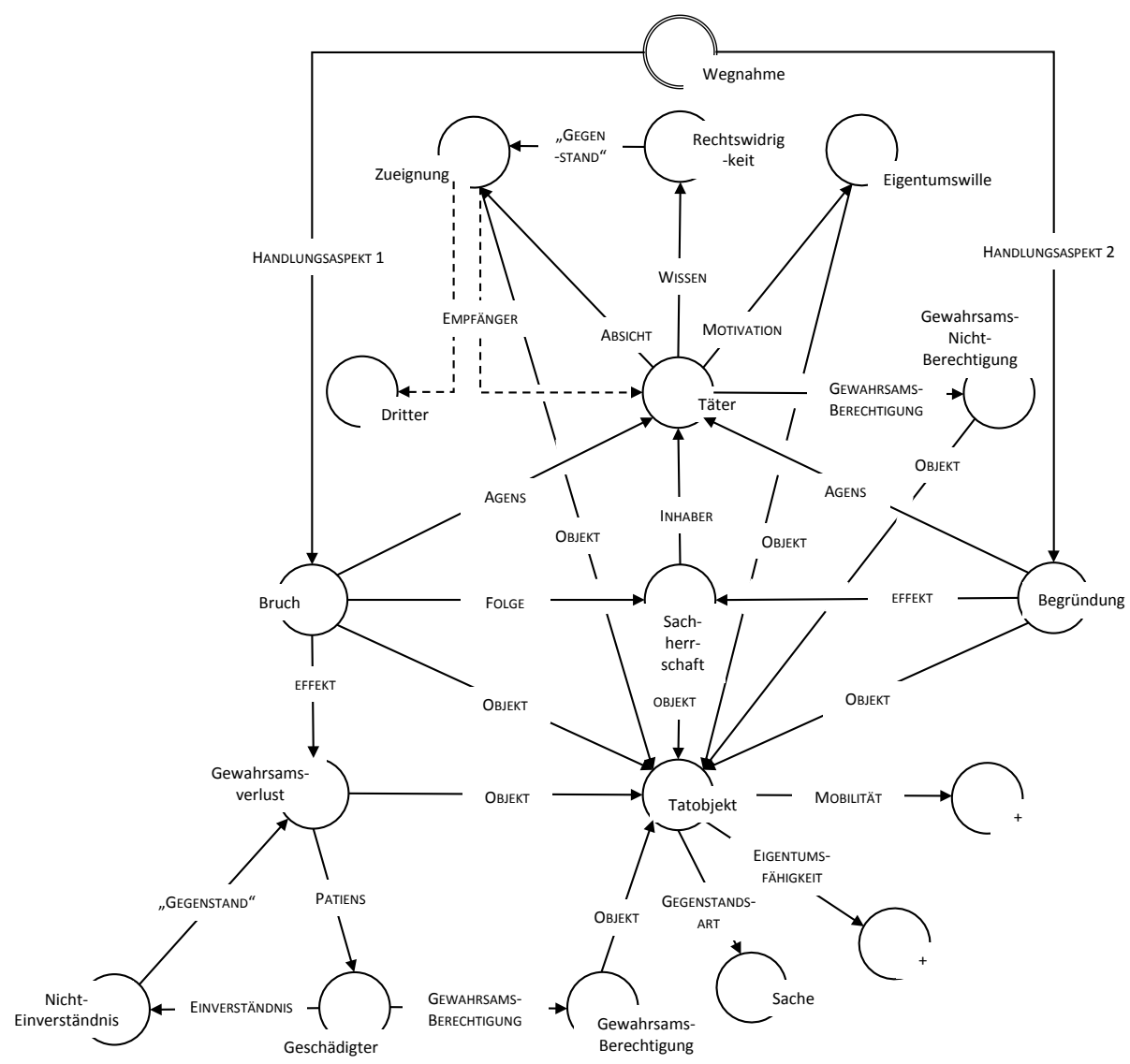

Abb. 9: Vollständiger Frame des Diebstahl-Begriffs nach § 242 StGB

\section{$7 \quad$ Fazit}

Was in der Gesamtschau der Modellierung deutlich zutage tritt, ist die zentrale Stellung des Tatobjekts innerhalb der im Frame modellierten Relation: Das Tatobjekt ist dasjenige Element, auf das bei weitem die meisten Attribut-Pfeile zu- 
laufen. So ist das Tatobjekt nicht nur Objekt der (durch die Wegnahme beabsichtigten) Zueignung und Objekt seines Eigentumswillens (um dessentwillen er die Wegnahme ausführt); es ist des Weiteren:

- Objekt des vom Täter ausgeführten Gewahrsamsbruchs sowie seiner Gewahrsamsbegründung,

- Objekt der Sachherrschaft (deren Inhaber der Täter durch die Gewahrsamsbegründung ist),

- Objekt des (durch den Bruch verursachten) Gewahrsamsverlusts seitens des Geschädigten und schließlich

- Objekt der Gewahrsamsberechtigung seitens des Geschädigten sowie Objekt der nicht gegebenen Gewahrsamsberechtigung seitens des Täters.

Das Tatobjekt steht allerdings noch zu einem weiteren Wegnahme-Aspekt in Beziehung: nämlich dem Umstand, dass es von einer Person auf die andere übergeht. Dieser Aspekt, den die Kommentarliteratur, wie schon oben erwähnt, unter dem Stichwort der "Gewahrsamsverschiebung" verhandelt, stellt gewissermaßen den ,realweltlichen Kern' des Wegnahme-Geschehens dar: Die Wegnahme bewirkt, dass jemand, der eine Sache zuvor in Gewahrsam hatte, sie jetzt nicht mehr in Gewahrsam hat, und an seiner Stelle nun der Wegnehmende Gewahrsamsinhaber ist. Dieser dynamische Aspekt, der für das Wegnahme-Konzept zwar insofern von Bedeutung ist, als ohne ihn nicht von Wegnahme gesprochen werden könnte, kommt im Frame allerdings nur indirekt, durch den resultativen Charakter der Effekt- bzw. Folge-Attribute zum Ausdruck: Nur daraus, dass die täterseitige Sachherrschaft als Folge-Aspekt der Bruch-Handlung modelliert ist, welche (zuvor) einen Gewahrsamsverlust bewirkt hat, lässt sich erschließen, dass der Gewahrsam an der Sache vom Geschädigten auf den Täter übergegangen ist.

Es sollte jedoch klar geworden sein, dass eine Modellierung des Rechtsbegriffs ,Wegnahme' nicht auf der Ebene des ,realweltlichen' Übergangs einer Sache von einer Person auf die andere stehen bleiben kann, denn aus dem Gewahrsamswechsel selbst lässt sich zunächst überhaupt nichts für das Diebstahl-Konzept Relevantes ableiten: Denn dass eine Sache zuerst in A's, und anschließend in B's Gewahrsam ist, könnte ebenso gut darauf beruhen, dass B die Sache gekauft hat, dass sie ihm geschenkt wurde oder dass sie aus sonstigen Gründen in seinen Gewahrsam gekommen ist. Für die framesemantische Modellierung wesentlich relevanter als der Gewahrsamswechsel selbst sind darum diejenigen Aspekte, die ihn zu einer Wegnahme im strafrechtlichen Sinne werden lassen. Und auch die 
hierfür in Anspruch genommenen Teil-Konzepte ,Bruch' und ,Begründung lassen sich nicht auf ,realweltliche" Handlungen wie bspw. ,eine Sache ergreifen und einstecken " herunterbrechen; vielmehr erhellt sich ihr Sinn erst dadurch, dass sie diese Handlungen und die dahinter stehenden Motive und Intentionen in einen rechtlich-normativen Rahmen einbetten - durch den sie dann als rechtwidrig und strafrechtlich sanktionierbar ausgewiesen werden können.

\section{Literatur}

Barsalou, Lawrence W. (1992): Frames, concepts, and conceptual fields. In: Lehrer, Adrienne / Kittay, Eva. F. (eds.): Frames, Fields, and Contrasts. Hillsdale NJ: Lawrence Erlbaum, 21-71.

Busse, Dietrich (2002): Bedeutungsfeststellung, Interpretation, Arbeit mit Texten? Juristische Auslegungstätigkeit in linguistischer Sicht. In: Haß-Zumkehr, Ulrike (Hrsg.): Sprache und Recht. (= Institut für deutsche Sprache, Jahrbuch 2001). Berlin / New York: de Gruyter, 136-162.

Busse, Dietrich (2015a): Begriffsstrukturen und die Beschreibung von Begriffswissen. Analysemodelle und -verfahren einer wissensanalytisch ausgerichteten Semantik (am Beispiel von Begriffen aus der Domäne Recht). In: Archiv für Begriffsgeschichte 56. 153-196.

Busse, Dietrich (2015b): Juristisches Wissen als institutionelle Begriffsstrukturen. Analyseansätze aus Kognitionswissenschaften und wissensanalytischer Semantik (am Beispiel von Gesetzes-Begriffen). In: Archiv für Rechts- und Sozialphilosophie 101/3. 354-385.

Busse, Dietrich / Felden, Michaela / Wulf, Detmer (2018): Bedeutungs- und Begriffswissen im Recht. Frame-Analysen von Rechtsbegriffen im Deutschen. Berlin / Boston: de Gruyter.

Fillmore, Charles J. (1968): The Case for Case. In: Emmon Bach / Robert T. Harms (eds.): Universals in Linguistic Theory. New York: Holt, Rinehart \& Winston 1968, 1-88.

Fillmore, Charles J. (1985): Frames and the Semantics of Understanding. In: Quaderni di Semantica 6, 222-254. 
Fillmore, Charles J. (2007): Valency Issues in FrameNet. In: Herbst, Thomas / Götz-Votteler, Katrin (eds.): Valency - theoretical, descriptive and cognitive issues. Berlin/New York: Mouton de Gruyter, 129-160.

Löbner, Sebastian (2015): Functional Concepts and Frames. In: Gamerschlag, Thomas / Gerland, Doris / Osswald, Rainer / Petersen, Wiebke (eds.): Meaning, Frames, and Conceptual Representation. Düsseldorf: Düsseldorf University Press, 15-42.

Minsky, Marvin (1975): A Framework for representing knowledge. In: Winston, P. H. (ed.), The Psychology of Computer Vision. New York: McGraw-Hill, 211-277.

Minsky, Marvin (1985): The Society of Mind. New York: Simon \& Schuster.

Petersen, Wiebke (2007): Representations of concepts as frames. In: The Baltic International Yearbook of Cognition, Logic and Communication (Vol. 2), 151-169.

\section{Verwendete Kommentare}

Fischer, Thomas (2011): Strafgesetzbuch und Nebengesetze (Beck'sche KurzKommentare Band 10). 58. Auflage. München: C. H. Beck.

Joecks, Wolfgang (2010): Strafgesetzbuch: Studienkommentar. 9. Auflage. München: C. H. Beck.

Joecks, Wolfgang / Miebach, Klaus (Hrsg.) (2012): Münchener Kommentar zum Strafgesetzbuch [MüKo]. Band 3: §§ 185-262 StGB. 2. Auflage. München: C. H. Beck.

Laufhütte, Heinrich Wilhelm / Rissing-van Saan, Ruth / Tiedemann, Klaus (Hrsg.) 2010: Leipziger Kommentar. Strafgesetzbuch [LK]. Band 8: §§ 242-262 StGB. 12. Auflage. Berlin: de Gruyter.

Säcker, Franz Jürgen / Rixecker, Rolang (Hrsg.) (2012): Münchener Kommentar zum Bürgerlichen Gesetzbuch [MüKo]. Band 2: §§ 241-432. 6. Auflage. München: C. H. Beck.

Schönke, Adolf / Schröder, Horst (Hrsg.) (2010): Strafgesetzbuch. Kommentar. 28. Auflage. München: C. H. Beck.

Staudinger, Julius von (2012): Eckpfeiler des Zivilrechts. Neubearbeitung 2012. Berlin: Sellier / de Gruyter. 